\title{
Heat kernel asymptotics with mixed boundary conditions
}

\author{
Thomas P. Branson, Peter B. Gilkey*, \\ Klaus Kirsten† and Dmitri V. Vassilevich ${ }^{\ddagger}$
}

August 15, 2018

\begin{abstract}
We calculate the coefficient $a_{5}$ of the heat kernel asymptotics for an operator of Laplace type with mixed boundary conditions on a general compact manifold.
\end{abstract}

\section{Introduction}

Let $V$ be a vector bundle over a smooth compact Riemannian manifold $M$ of dimension $m$ with smooth boundary $\partial M$. Let $D$ be a second order operator of Laplace type on $C^{\infty}(V)$; this means the leading symbol of $D$ is given by the metric tensor, or equivalently, that $D$ has the form given in equations (11) and (2) below. Note that many natural second order operators which arise in applications are of Laplace type. If the boundary is non-empty, then we must impose suitable boundary conditions. We assume given a decomposition $\left.V\right|_{\partial M}=V_{N} \oplus V_{D}$; extend the decomposition to be parallel with respect to the normal geodesic rays on a collared neighborhood of the boundary. Let $S$ be an auxiliary endomorphism of the bundle $V_{N}$ and let $\phi_{; m}$

${ }^{*}$ Research partially supported by the ESI (Austria), IHES (France), and the NSF (USA)

${ }^{\dagger}$ Research partially supported by the DFG under contract number Bo1112/4-2 and the EPSRC under Grant No GR/M45726

${ }^{\ddagger}$ Research partially supported by the Alexander von Humboldt foundation (Germany), ICTP (Italy) and GRACENAS (Russia) 
be the covariant derivative of $\phi \in C^{\infty}(V)$ with respect to the inward unit normal. Decompose $\phi=\phi^{N} \oplus \phi^{D}$ and set

$$
\mathcal{B} \phi:=\left.\left.\phi^{D}\right|_{V_{D}} \oplus\left(\phi_{; m}^{N}+S \phi^{N}\right)\right|_{V_{N}} .
$$

Dirichlet boundary conditions correspond to vanishing $V_{N}$; Robin (i.e. modified Neumann) boundary conditions correspond to vanishing $V_{D}$. Let $D_{\mathcal{B}}$ be the realization of $D ; \phi \in \operatorname{Domain}\left(D_{\mathcal{B}}\right)$ if and only if $\mathcal{B} \phi=0$. This is an elliptic boundary value problem and satisfies the Lopatinski-Shapiro condition.

Let $F$ be an auxiliary smooth function on $M$, for use in localization or 'smearing'. Let $e^{-t D_{\mathcal{B}}}$ be the fundamental solution of the heat equation; this is trace class on $L^{2}(V)$ and as $t \downarrow 0$, there is an asymptotic expansion:

$$
\operatorname{Tr}_{L^{2}}\left(F e^{-t D_{\mathcal{B}}}\right) \cong \sum_{n \geq 0} t^{(n-m) / 2} a_{n}(F, D, \mathcal{B})
$$

see [11, 12, 16] for details. The invariants $a_{n}(F, D, \mathcal{B})$ are locally computable in terms of geometric invariants and form the focus of our study.

The study of these invariants can be physically motivated. Consider a field theory in which $D$ defines the quadratic form of the action; see, for example [9]. We note that consistent local boundary conditions for fields of non-zero spin are inevitably mixed ones. The one-loop effective action is (formally) given by $\log \operatorname{det}(D)$. The heat kernel coefficients up to $a_{m}$ describe ultraviolet divergencies of the theory. The coefficient $a_{m}$ also defines the scale anomaly. The leading term in the large mass expansion of the effective action is given by $a_{m+1}$; the lower coefficients are usually absorbed in renormalizations. Variations of the heat kernel coefficients give vacuum expectation values of currents, see [4] for example. The study of these coefficients is important for renormalization and calculation of anomalies in higher dimensional models [17], and for large mass expansion and calculation of currents in four dimensions.

McKean and Singer [14] studied the invariants $a_{0}, a_{1}$, and $a_{2}$. Kennedy et al. [10] studied the invariant $a_{3}$. Moss and Dowker [15] studied $a_{4}$ for the scalar Laplacian. Branson and Gilkey [1] determined $a_{4}$ in the vector valued case; a minor error in the calculation of two of the coefficients was later corrected by Vassilevich [18]. These results are summarized in Theorem 2.1. Many other authors have also studied these coefficients.

We say the boundary conditions are pure if $V_{N}$ or $V_{D}$ vanishes. The coefficient $a_{5}$ has been determined previously for pure boundary conditions. Branson, Gilkey and Vassilevich [3] studied the special cases of a domain $M$ in flat space and of a curved domain with totally geodesic boundary. Kirsten [13 generalized these results to arbitrary manifolds and boundaries; see also Dowker and Kirsten [6] for related work. We summarize the results of those 
papers in Lemmas 2.2 and 2.3. The main result of this paper is Theorem 2.4 which gives $a_{5}$ for general mixed boundary conditions.

We have decoupled the calculation of $a_{5}$ in the general setting into three pieces. The first piece, $\mathcal{A}_{5}^{1}$, was computed in [3]; it gives $a_{5}$ for pure boundary conditions if the boundary is totally geodesic. The second piece $\mathcal{A}_{5}^{2}$ was computed in [13]; it contains the terms involving the second fundamental form which are necessary to deal with the case where the boundary is not totally geodesic [13. The final piece $\mathcal{A}_{5}^{3}$ is computed in this paper; it contains the additional 42 terms which describe the interaction of $V_{N}$ and $V_{D}$ for general mixed boundary conditions. Our purpose in this paper is not purely combinatorial; determining these additional coefficients requires us to derive new functorial properties of the invariants which are important in their own right.

In $\$ 3$, we use invariance theory to show that the computation of $a_{5}$ can be decomposed into the three pieces discussed above and to show that the undetermined coefficients $w_{i}$ describing the interaction of $V_{N}$ and $V_{D}$ are universal coefficients which are dimension free. The remainder of the paper is devoted to computing these coefficients; see the cross reference table following Theorem 2.4. We postpone until Appendices A, B, and $\mathrm{C}$ the discussion of some combinatorial formulas we shall need.

We use three different technical tools in the proof of Theorem 2.4.

1. The index theorem. Let $P: C^{\infty}(V) \rightarrow C^{\infty}(W)$ be an elliptic complex of Dirac type. Impose suitable boundary conditions. Let $D:=P^{*} P$ and $\hat{D}:=P P^{*}$ be the associated operators of Laplace type. An observation of Bott shows that with suitable boundary conditions $a_{m}(1, D, \mathcal{B})-a_{m}(1, \hat{D}, \mathcal{B})=\operatorname{index}(P)$ and $a_{n}(1, D, \mathcal{B})-a_{n}(1, \hat{D}, \mathcal{B})=0$ if $n \neq m$; this is often called the local index formula. We shall apply this observation in $\S$ a and in $\S[7$.

2. Conformal variations. Let $D(\epsilon)=e^{-2 \epsilon F} D$. We then have that $\left.\frac{d}{d \epsilon}\right|_{\epsilon=0} a_{n}(1, D, \mathcal{B})=(m-n) a_{n}(F, D, \mathcal{B})$. Furthermore, if $m=n+2$, then $\left.\frac{d}{d \epsilon}\right|_{\epsilon=0} a_{n}\left(e^{-2 \epsilon f} F, e^{-2 \epsilon f} D, \mathcal{B}\right)=0$. We use these identities in $\S 6$.

3. Calculations on the ball. The invariant $a_{n}$ was computed for the Laplacians on spinors and on 1-forms on the ball in [5, 7]; we use this computation in 85 .

\section{Statement of results}

If $D$ is an operator of Laplace type, then locally we have:

$$
D=-\left(g^{\mu \nu} \partial_{\mu} \partial_{\nu}+A^{\sigma} \partial_{\sigma}+B\right) \text {. }
$$


Here $g^{\mu \nu}$ acts by scalar multiplication; $A^{\sigma}$ and $B$ are matrix valued. We can also express $D$ covariantly. There is a unique connection $\nabla$ on $V$ and a unique endomorphism $E$ of $V$ so that

$$
D=-\left(\operatorname{Tr}\left(\nabla^{2}\right)+E\right)
$$

Let $\omega$ be the local 1 -form of the connection $\nabla$. We may express:

$$
\begin{aligned}
& \omega_{\delta}=\frac{1}{2} g_{\nu \delta}\left(A^{\nu}+g^{\mu \sigma} \Gamma_{\mu \sigma}^{\nu} I_{V}\right) \text { and } \\
& E=B-g^{\nu \mu}\left(\partial_{\mu} \omega_{\nu}+\omega_{\nu} \omega_{\mu}-\omega_{\sigma} \Gamma_{\nu \mu}{ }^{\sigma}\right) .
\end{aligned}
$$

If $D$ is the scalar Laplacian, then $\nabla$ is the flat connection and $E=0$. If $D=d \delta+\delta d$ on the bundle of $p$ forms, then $\nabla$ is Levi-Civita connection and the endomorphism $E$ is given in terms of curvature by the Weitzenböck formulas. Let $\tau$ be the scalar curvature. If $D$ is the spin Laplacian, then $\nabla$ is the spin connection, and the Lichnerowicz formula gives $E=-\frac{1}{4} \tau$.

We adopt the following notational conventions to express the asymptotic coefficients $a_{n}$ in terms of geometrical invariants. Let Roman indices $i, j, k$, and $l$ range from 1 through the dimension $m$ of the manifold and index a local orthonormal frame $\left\{e_{1}, \ldots, e_{m}\right\}$ for the tangent bundle of the manifold. Let Roman indices $a, b, c$, and $d$ range from 1 through $m-1$ and index a local orthonormal frame for the tangent bundle of the boundary $\partial M$. On the boundary, we shall let $e_{m}$ be the inward unit normal vector field. We shall not introduce bundle indices explicitly. Let $\operatorname{Tr}(\mathcal{A})$ be the fiber trace of an endomorphism $\mathcal{A}$ of $V$. Greek indices will index coordinate frames. We adopt the Einstein convention and sum over repeated indices.

Let $R_{i j k l}$ be the components of the curvature tensor of the Levi-Civita connection, let $\rho_{i j}:=R_{i k k j}$ be the components of the Ricci tensor, and let $\tau:=\rho_{i i}$ be the scalar curvature. With our sign convention, $R_{1212}=-1$ on the unit sphere $S^{2}$ in Euclidean space. Let $\Omega_{i j}$ be the endomorphism-valued components of the curvature of the connection $\nabla$ on $V$. Let $\Gamma$ be the Christoffel symbols of the Levi-Civita connection. Let $L_{a b}:=\left(\nabla_{e_{a}} e_{b}, e_{m}\right)=\Gamma_{a b m}$ give the second fundamental form. We use the Levi-Civita connections and the connection $\nabla$ to covariantly differentiate tensors of all types. Let ';' denote multiple covariant differentiation with respect to the Levi-Civita connection of $M$ and let ':' denote multiple tangential covariant differentiation on the boundary with respect to the Levi-Civita connection of the boundary; the difference between ';' and ':' is measured by the second fundamental form. Thus, for example, $E_{; a}=E_{: a}$ since there are no tangential indices in $E$ to be differentiated. On the other hand, $E_{; a b} \neq E_{: a b}$ since the index $a$ is also being differentiated. Since $L$ and $S$ are only defined on the boundary, these tensors can only be differentiated tangentially. Let $d x$ and $d y$ be the Riemannian 
volume elements on $M$ and on $\partial M$ respectively. Let $f_{1} \in C^{\infty}(M)$ and let $f_{2} \in C^{\infty}(\partial M)$. Let

$$
f_{1}[M]:=\int_{M} f_{1}(x) d x \text { and } f_{2}[\partial M]=\int_{\partial M} f_{2}(y) d y .
$$

Let $\Pi_{-}$be orthogonal projection on $V_{D}$ and let $\Pi_{+}$be orthogonal projection on $V_{N}$. Let $\chi:=\Pi_{+}-\Pi_{-} ; \chi=+1$ on $V_{N}$ and $\chi=-1$ on $V_{D}$. Extend $\chi$ to be parallel along normal geodesic rays. We refer to [1] for the proof of the following result:

\section{Theorem 2.1}

$$
\begin{aligned}
& \text { 1. } a_{0}(F, D, \mathcal{B})=(4 \pi)^{-m / 2} \operatorname{Tr}(F)[M] \text {. } \\
& \text { 2. } a_{1}(F, D, \mathcal{B})=\frac{1}{4}(4 \pi)^{-(m-1) / 2} \operatorname{Tr}(\chi F)[\partial M] \text {. } \\
& \text { 3. } a_{2}(F, D, \mathcal{B})=\frac{1}{6}(4 \pi)^{-m / 2}\{\operatorname{Tr}(6 F E+F \tau)[M] \\
& \left.+\operatorname{Tr}\left(2 F L_{a a}+3 \chi F_{; m}+12 F S\right)[\partial M]\right\} . \\
& \text { 4. } a_{3}(F, D, \mathcal{B})=\frac{1}{384}(4 \pi)^{-(m-1) / 2} \operatorname{Tr}\left\{F \left(96 \chi E+16 \chi \tau+8 F \chi R_{\text {amam }}\right.\right. \\
& +\left(13 \Pi_{+}-7 \Pi_{-}\right) L_{a a} L_{b b}+\left(2 \Pi_{+}+10 \Pi_{-}\right) L_{a b} L_{a b}+96 S L_{a a}+192 S^{2} \\
& \left.\left.-12 \chi_{: a} \chi_{: a}\right)+F_{; m}\left(\left(6 \Pi_{+}+30 \Pi_{-}\right) L_{a a}+96 S\right)+24 \chi F_{; m m}\right\}[\partial M] . \\
& \text { 5. } a_{4}(F, D, \mathcal{B})=\frac{1}{360}(4 \pi)^{-m / 2}\left\{\operatorname { T r } \left\{F \left(60 E_{; i i}+60 \tau E+180 E^{2}\right.\right.\right. \\
& \left.\left.+30 \Omega_{i j} \Omega_{i j}+12 \tau_{; i i}+5 \tau^{2}-2 \rho_{i j} \rho_{i j}+2 R_{i j k l} R_{i j k l}\right)\right\}[M] \\
& +\operatorname{Tr}\left\{F \left\{\left(240 \Pi_{+}-120 \Pi_{-}\right) E_{; m}+\left(42 \Pi_{+}-18 \Pi_{-}\right) \tau_{; m}\right.\right. \\
& +24 L_{a a: b b}+0 L_{a b: a b}+120 E L_{a a}+20 \tau L_{a a}+4 R_{a m a m} L_{b b} \\
& -12 R_{a m b m} L_{a b}+4 R_{a b c b} L_{a c}+0 \Omega_{a m: a}+\frac{1}{21}\left\{\left(280 \Pi_{+}+40 \Pi_{-}\right) L_{a a} L_{b b} L_{c c}\right. \\
& \left.+\left(168 \Pi_{+}-264 \Pi_{-}\right) L_{a b} L_{a b} L_{c c}+\left(224 \Pi_{+}+320 \Pi_{-}\right) L_{a b} L_{b c} L_{a c}\right\} \\
& +720 S E+120 S \tau+0 S R_{a m a m}+144 S L_{a a} L_{b b}+48 S L_{a b} L_{a b} \\
& +480 S^{2} L_{a a}+480 S^{3}+120 S_{: a a}+60 \chi \chi_{: a} \Omega_{a m}-12 \chi_{: a} \chi_{: a} L_{b b} \\
& \left.-24 \chi_{: a} \chi_{: b} L_{a b}-120 \chi_{: a} \chi_{: a} S\right\}+F_{; m}\left(180 \chi E+30 \chi \tau+0 R_{a m a m}\right. \\
& +\frac{1}{7}\left\{\left(84 \Pi_{+}-180 \Pi_{-}\right) L_{a a} L_{b b}+\left(84 \Pi_{+}+60 \Pi_{-}\right) L_{a b} L_{a b}\right\} \\
& \left.+72 S L_{a a}+240 S^{2}-18 \chi_{: a} \chi_{: a}\right)+F_{; m m}\left(24 L_{a a}+120 S\right) \\
& \left.\left.+30 \chi F_{; i i m}\right\}[\partial M]\right\} \text {. }
\end{aligned}
$$

We introduce some additional notation to discuss $a_{5}$. Let: 


$$
\begin{aligned}
\mathcal{A}_{5}^{1}:= & F\left\{360 \chi E_{; m m}+1440 E_{; m} S\right. \\
& +720 \chi E^{2}+240 \chi E_{: a a}+240 \chi \tau E+48 \chi \tau_{; i i}+20 \chi \tau^{2} \\
& -8 \chi \rho_{i j} \rho_{i j}+8 \chi R_{i j k l} R_{i j k l}-120 \chi \rho_{m m} E-20 \chi \rho_{m m} \tau \\
& +480 \tau S^{2}+12 \chi \tau_{; m m}+24 \chi \rho_{m m: a a}+15 \chi \rho_{m m ; m m} \\
& +270 \tau_{; m} S+120 \rho_{m m} S^{2}+960 S S_{: a a}+16 \chi R_{a m m b} \rho_{a b} \\
& \left.-17 \chi \rho_{m m} \rho_{m m}-10 \chi R_{a m m b} R_{a m m b}+2880 E S^{2}+1440 S^{4}\right\} \\
+ & F_{; m}\left\{\left(\frac{195}{2} \Pi_{+}-60 \Pi_{-}\right) \tau_{; m}+240 \tau S-90 \rho_{m m} S+270 S_{: a a}\right. \\
& \left.+\left(630 \Pi_{+}-450 \Pi_{-}\right) E_{; m}+1440 E S+720 S^{3}\right\} \\
+ & F_{; m m}\left\{60 \chi \tau-90 \chi \rho_{m m}+360 \chi E+360 S^{2}\right\} \\
+ & 180 S F_{; m m m}+45 \chi F_{; m m m m} .
\end{aligned}
$$

Let $\mathcal{B}_{S}^{-}$denote pure Dirichlet boundary conditions if $V_{N}$ vanishes; $S$ plays no role here. Similarly let $\mathcal{B}_{S}^{+}$denote Neumann boundary conditions modified by $S$ if $V_{D}$ vanishes. The following result was proved in [3].

Lemma 2.2 If the boundary of $M$ is totally geodesic, then we have

$$
\begin{gathered}
a_{5}\left(F, D, \mathcal{B}_{S}^{ \pm}\right)=\frac{1}{5760}(4 \pi)^{-(m-1) / 2} \operatorname{Tr}\left\{\mathcal{A}_{5}^{1}+120 F \chi \Omega_{a b} \Omega_{a b}\right. \\
\left.+\left(90 \Pi_{+}+360 \Pi_{-}\right) F \Omega_{a m} \Omega_{a m}+600 F S_{a} S_{: a}\right\}[\partial M]
\end{gathered}
$$

Next we introduce terms involving the second fundamental form $L$. Let:

$$
\begin{aligned}
\mathcal{A}_{5}^{2}:= & F\left\{\left(90 \Pi_{+}+450 \Pi_{-}\right) L_{a a} E_{; m}+\left(\frac{111}{2} \Pi_{+}+42 \Pi_{-}\right) L_{a a} \tau_{; m}\right. \\
& +30 \Pi_{+} L_{a b} R_{a m m b ; m}+240 L_{a a} S_{: b b}+420 L_{a b} S_{: a b}+390 L_{a a: b} S_{: b} \\
& +480 L_{a b: a} S_{: b}+420 L_{a a: b b} S+60 L_{a b: a b} S \\
& +\left(\frac{487}{16} \Pi_{+}+\frac{413}{16} \Pi_{-}\right) L_{a a: b} L_{c c: b}+\left(238 \Pi_{+}-58 \Pi_{-}\right) L_{a b: a} L_{c c: b} \\
& +\left(\frac{49}{4} \Pi_{+}+\frac{11}{4} \Pi_{-}\right) L_{a b: a} L_{b c: c}+\left(\frac{535}{8} \Pi_{+}-\frac{355}{8} \Pi_{-}\right) L_{a b: c} L_{a b: c} \\
& +\left(\frac{151}{4} \Pi_{+}+\frac{29}{4} \Pi_{-}\right) L_{a b: c} L_{a c: b}+\left(111 \Pi_{+}-6 \Pi_{-}\right) L_{a a: b b} L_{c c} \\
& +\left(-15 \Pi_{+}+30 \Pi_{-}\right) L_{a b: a b} L_{c c}+\left(-\frac{15}{2} \Pi_{+}+\frac{75}{2} \Pi_{-}\right) L_{a b: a c} L_{b c} \\
& +\left(\frac{945}{4} \Pi_{+}-\frac{285}{4} \Pi_{-}\right) L_{a a: b c} L_{b c}+\left(114 \Pi_{+}-54 \Pi_{-}\right) L_{b c: a a} L_{b c} \\
& +1440 L_{a a} S E+30 L_{a a} S \rho_{m m}+240 L_{a a} S \tau-60 L_{a b} \rho_{a b} S \\
& +180 L_{a b} S R_{a m m b}+\left(195 \Pi_{+}-105 \Pi_{-}\right) L_{a a} L_{b b} E \\
& +\left(30 \Pi_{+}+150 \Pi_{-}\right) L_{a b} L_{a b} E+\left(\frac{195}{6} \Pi_{+}-\frac{105}{6} \Pi_{-}\right) L_{a a} L_{b b} \tau \\
& +\left(5 \Pi_{+}+25 \Pi_{-}\right) L_{a b} L_{a b} \tau+\left(-\frac{275}{16} \Pi_{+}+\frac{215}{16} \Pi_{-}\right) L_{a a} L_{b b} \rho_{m m} \\
& +\left(-\frac{275}{8} \Pi_{+}+\frac{215}{8} \Pi_{-}\right) L_{a b} L_{a b} \rho_{m m}+\left(-\Pi_{+}-14 \Pi_{-}\right) L_{c c} L_{a b} \rho_{a b} \\
& +\left(\frac{109}{4} \Pi_{+}-\frac{49}{4} \Pi_{-}\right) L_{c c} L_{a b} R_{a m m b}+16 \chi L_{a b} L_{a c} \rho_{b c} \\
& +\left(\frac{133}{2} \Pi_{+}+\frac{47}{2} \Pi_{-}\right) L_{a b} L_{a c} R_{b m m c}-32 \chi L_{a b} L_{c d} R_{a c b d} \\
& +\frac{315}{2} L_{c c} L_{a b} L_{a b} S+\left(\frac{2041}{128} \Pi_{+}+\frac{65}{128} \Pi_{-}\right) L_{a a} L_{b b} L_{c c} L_{d d}
\end{aligned}
$$




$$
\begin{aligned}
& +150 L_{a b} L_{b c} L_{a c} S+\left(\frac{417}{32} \Pi_{+}+\frac{141}{32} \Pi_{-}\right) L_{c c} L_{d d} L_{a b} L_{a b} \\
& +1080 L_{a a} L_{b b} S^{2}+360 L_{a b} L_{a b} S^{2}+\left(\frac{375}{32} \Pi_{+}-\frac{777}{32} \Pi_{-}\right) L_{a b} L_{a b} L_{c d} L_{c d} \\
& +\frac{885}{4} L_{a a} L_{b b} L_{c c} S+\left(25 \Pi_{+}-\frac{17}{2} \Pi_{-}\right) L_{d d} L_{a b} L_{b c} L_{a c} \\
& \left.+2160 L_{a a} S^{3}+\left(\frac{231}{8} \Pi_{+}+\frac{327}{8} \Pi_{-}\right) L_{a b} L_{b c} L_{c d} L_{d a}\right\} \\
+ & F_{; m}\left\{\left(90 \Pi_{+}+450 \Pi_{-}\right) L_{a a} E+\left(-\frac{165}{8} \Pi_{+}-\frac{255}{8} \Pi_{-}\right) L_{a a} \rho_{m m}\right. \\
& +\left(15 \Pi_{+}+75 \Pi_{-}\right) L_{a a} \tau+600 L_{a a} S^{2}+\left(\frac{1215}{8} \Pi_{+}-\frac{315}{8} \Pi_{-}\right) L_{a a: b b} \\
& -\frac{45}{4} \chi L_{a b: a b}+\left(15 \Pi_{+}-30 \Pi_{-}\right) L_{a b} \rho_{a b} \\
& +\left(-\frac{165}{4} \Pi_{+}+\frac{465}{4} \Pi_{-}\right) L_{a b} R_{a m m b}+\frac{705}{4} L_{a a} L_{b b} S-\frac{75}{2} L_{a b} L_{a b} S \\
& +\left(\frac{459}{32} \Pi_{+}+\frac{495}{32} \Pi_{-}\right) L_{a a} L_{b b} L_{c c}+\left(\frac{267}{16} \Pi_{+}-\frac{1485}{16} \Pi_{-}\right) L_{c c} L_{a b} L_{a b} \\
& \left.+\left(-54 \Pi_{+}+\frac{225}{2} \Pi_{-}\right) L_{a b} L_{b c} L_{a c}\right\} \\
+ & F_{; m m}\left\{30 L_{a a} S+\left(\frac{315}{16} \Pi_{+}-\frac{1215}{16} \Pi_{-}\right) L_{a a} L_{b b}\right. \\
& +\left(-\frac{645}{8} \Pi_{+}+\frac{945}{8} \Pi_{-}\right) L_{a b} L_{a b} \\
+ & F_{; m m m}\left(-30 \Pi_{+}+105 \Pi_{-}\right) L_{a a} .
\end{aligned}
$$

The following result was proved in [13].

Lemma 2.3 We have:

$$
\begin{aligned}
& a_{5}\left(F, D, \mathcal{B}_{S}^{ \pm}\right)=\frac{1}{5760}(4 \pi)^{-(m-1) / 2} \operatorname{Tr}\left\{\mathcal{A}_{5}^{1}+\mathcal{A}_{5}^{2}+120 F \chi \Omega_{a b} \Omega_{a b}\right. \\
& \left.+\left(90 \Pi_{+}+360 \Pi_{-}\right) F \Omega_{a m} \Omega_{a m}+600 F S_{: a} S_{: a}\right\}[\partial M] .
\end{aligned}
$$

To generalize Lemma 2.3 to mixed boundary conditions, we introduce the additional terms describing the interaction of $V_{N}$ and $V_{D}$. Let:

$$
\begin{aligned}
\mathcal{A}_{5}^{3}(\vec{w}) & :=F\left\{w_{1} E^{2}+w_{2} \chi E \chi E+w_{3} S_{: a} S_{: a}+w_{4} \chi S_{: a} S_{: a}\right. \\
& +w_{5} \Omega_{a b} \Omega_{a b}+w_{6} \chi \Omega_{a b} \Omega_{a b}+w_{7} \chi \Omega_{a b} \chi \Omega_{a b}+w_{8} \Omega_{a m} \Omega_{a m} \\
& +w_{9} \chi \Omega_{a m} \Omega_{a m}+w_{10} \chi \Omega_{a m} \chi \Omega_{a m}+w_{11}\left(\Omega_{a m} \chi S_{: a}-\Omega_{a m} S_{: a} \chi\right) \\
& +w_{12} \chi \chi_{: a} \Omega_{a m} L_{c c}+w_{13} \chi_{: a} \chi_{: b} \Omega_{a b}+w_{14} \chi \chi_{: a} \chi_{: b} \Omega_{a b} \\
& +w_{15} \chi \chi_{: a} \Omega_{a m ; m}+w_{16} \chi \chi_{: a} \Omega_{a b: b}+w_{17} \chi_{: a} \Omega_{b m} L_{a b}+w_{18} \chi_{: a} E_{: a} \\
& +w_{19} \chi_{: a} \chi_{: a} E+w_{20} \chi_{: a} \chi_{: a} E+w_{21} \chi_{: a a} E+w_{22} \chi_{: a} \chi_{: a} \tau \\
& +w_{23} \chi_{: a} \chi_{: a} \rho_{m m}+w_{24} \chi_{: a} \chi_{: b} \rho_{a b}+w_{25} \chi_{: a} \chi_{: b} R_{m a b m} \\
& +w_{26} \chi_{: a} \chi_{: a} L_{b b} L_{c c}+w_{27} \chi_{: a} \chi_{: b} L_{a c} L_{b c}+w_{28} \chi_{: a} \chi_{: a} L_{c d} L_{c d} \\
& +w_{29} \chi_{: a} \chi_{: b} L_{a b} L_{c c}+w_{30} \chi_{: a} S_{: a} L_{c c}+w_{31} \chi_{: a} S_{: b} L_{a b} \\
& +w_{32} \chi_{: a} \chi_{: a} \chi_{: b} \chi_{: b}+w_{33} \chi_{: a} \chi_{: b} \chi_{: a} \chi_{: b}+w_{34} \chi_{: a a} \chi_{: b b}+w_{35} \chi_{: a b} \chi_{: a b} \\
& \left.+w_{36} \chi_{: a} \chi_{: a} \chi_{: b b}+w_{37} \chi_{: b} \chi_{: a a b}\right\}+F_{; m}\left\{w_{38} \chi_{: a} S_{: a}+w_{39} \chi_{: a} \chi_{: a} L_{c c}\right. \\
& \left.+w_{40} \chi_{: a} \chi_{: b} L_{a b}+w_{41} \chi_{: a} \Omega_{a m}\right\}+F_{; m m}\left\{w_{42} \chi_{: a} \chi_{: a}\right\} .
\end{aligned}
$$


The following Theorem represents the main result of this paper:

Theorem 2.4 We have

$$
a_{5}(F, D, \mathcal{B})=\frac{1}{5760}(4 \pi)^{-(m-1) / 2} \operatorname{Tr}\left(\mathcal{A}_{1}^{1}+\mathcal{A}_{5}^{2}+\mathcal{A}_{5}^{3}(\vec{w})\right)[\partial M]
$$

where the universal constants $\vec{w}=\left(w_{1}, \ldots, w_{42}\right)$ are given by:

\begin{tabular}{|l|l|l|l|l|}
\hline$w_{1}=-180$ & $w_{2}=180$ & $w_{3}=-120$ & $w_{4}=720$ & $w_{5}=-\frac{105}{4}$ \\
\hline$w_{6}=120$ & $w_{7}=\frac{105}{4}$ & $w_{8}=-45$ & $w_{9}=180$ & $w_{10}=-45$ \\
\hline$w_{11}=360$ & $w_{12}=45$ & $w_{13}=-180$ & $w_{14}=90$ & $w_{15}=90$ \\
\hline$w_{16}=120$ & $w_{17}=180$ & $w_{18}=300$ & $w_{19}=-180$ & $w_{20}=-90$ \\
\hline$w_{21}=240$ & $w_{22}=-30$ & $w_{23}=0$ & $w_{24}=-60$ & $w_{25}=30$ \\
\hline$w_{26}=-\frac{675}{32}$ & $w_{27}=-\frac{75}{4}$ & $w_{28}=-\frac{195}{16}$ & $w_{29}=-\frac{675}{8}$ & $w_{30}=-330$ \\
\hline$w_{31}=-300$ & $w_{32}=\frac{15}{4}$ & $w_{33}=\frac{15}{8}$ & $w_{34}=-\frac{15}{4}$ & $w_{35}=-\frac{105}{2}$ \\
\hline$w_{36}=-15$ & $w_{37}=-\frac{135}{2}$ & $w_{38}=-210$ & $w_{39}=-\frac{165}{16}$ & $w_{40}=-\frac{405}{8}$ \\
\hline$w_{41}=135$ & $w_{42}=-30$ & & & \\
\hline
\end{tabular}

The remainder of this paper is devoted to the evaluation of these constants; the various coefficients are determined in the following sections:

\begin{tabular}{|c|c|c|c|c|c|c|c|c|c|c|c|}
\hline$w_{1}$ & 8 & & $\delta$ 国 & & $\oint \boxminus$ & & & & $\S \sqrt{0}$ & $w_{6}$ & \\
\hline$u_{7}$ & $8 \sqrt{7}$ & $w_{8}$ & $\delta 4$ & 9 & $\oint 4$ & $i_{10}$ & & $w_{1}$ & $\S \square$ & & \\
\hline$w_{1}$ & $8 \sqrt{7}$ & $w_{14}$ & $\oint \sqrt{0}$ & 15 & $\oint 4$ & $w_{1}$ & 6 & $w$ & $\S \sqrt{7}$ & & \\
\hline$w_{19}$ & 83 & $w_{20}$ & $\S \longdiv { 4 }$ & $w_{21}$ & 86 & $w_{22}$ & $8 \overline{3}$ & $w_{2}$ & $\S \sqrt{6}$ & $w_{2}$ & \\
\hline$w_{25}$ & 86 & $w_{26}$ & $\S 5$ & & 86 & $w_{2}$ & 9 & & $\$ 6$ & & \\
\hline$w_{31}$ & 86 & $w_{3}$ & 86 & $w_{33}$ & 86 & $w_{3}$ & 30 & & $\$ 6$ & & \\
\hline$v_{37}$ & 86 & $U_{38}$ & 6 & $v_{39}$ & $\$ 6$ & $w_{40}$ & $\S \overline{6}$ & $w_{4}$ & 0 & $w_{42}$ & \\
\hline
\end{tabular}

\section{Invariance theory}

The main result of this section is the following technical result.

Lemma 3.1 There exist universal constants $w_{i}$ so that

$$
a_{5}(F, D, \mathcal{B})=\frac{1}{5760}(4 \pi)^{-(m-1) / 2} \operatorname{Tr}\left(\mathcal{A}_{1}^{1}+\mathcal{A}_{5}^{2}+\mathcal{A}_{5}^{3}(\vec{w})\right)[\partial M] .
$$

Let $N^{\nu}(F)=F_{; m \ldots m}$ be the $\nu^{\text {th }}$ normal covariant derivative of the smearing function $F$. Let $R_{i j k l}, \Omega_{i j}$, and $E$ have order 2 ; let $L_{a b}$ and $S$ have order 1 , and let $\chi$ have order 0 . We increase the order by 1 for every explicit covariant derivative. The following Lemma is proved in [1] and summarizes some of the properties of the invariants $a_{n}$ that we shall need.

\section{Lemma 3.2}


1. There exists an invariant local formula $a_{n}^{M}=a_{n}^{M}(x, D)$ on $M$ which is homogeneous of order $n$ and which vanishes if $n$ is odd, and there exist invariant local formulas $a_{n, \nu}^{\partial M}=a_{n, \nu}^{\partial M}(y, D, \mathcal{B})$ on $\partial M$ which are homogeneous of order $n-\nu-1$ so that

$$
a_{n}(F, D, \mathcal{B})=\operatorname{Tr}\left(F a_{n}^{M}\right)[M]+\sum_{0 \leq \nu \leq n-1} \operatorname{Tr}\left(N^{\nu}(F) a_{n, \nu}^{\partial M}\right)[\partial M]
$$

2. Let $M=M_{1} \times M_{2}$ for $M_{1}$ closed. Let $D=D_{1} \otimes 1+1 \otimes D_{2}$. Then

$$
\begin{aligned}
& a_{n}^{M}(x, D)=\sum_{p+q=n} a_{p}^{M_{1}}\left(x_{1}, D_{1}\right) a_{q}^{M_{2}}\left(x_{2}, D_{2}\right) \text { and } \\
& a_{n, \nu}^{\partial M}(y, D, \mathcal{B})=\sum_{p+q=n} a_{p}^{M_{1}}\left(x_{1}, D_{1}\right) a_{q, \nu}^{\partial M_{2}}\left(y_{2}, D_{2}, \mathcal{B}\right) .
\end{aligned}
$$

3. Let $D=D_{1} \oplus D_{2}$ over $M$. Then

$$
\begin{aligned}
& a_{n}^{M}(x, D)=a_{n}^{M}\left(x, D_{1}\right)+a_{n}^{M}\left(x, D_{2}\right) \text { and } \\
& a_{n, \nu}^{\partial M}(y, D, \mathcal{B})=a_{n, \nu}^{\partial M}\left(y, D_{1}, \mathcal{B}\right)+a_{n, \nu}^{\partial M}\left(y, D_{2}, \mathcal{B}\right) .
\end{aligned}
$$

4. We have the following variational formulas:

$$
\begin{aligned}
& \left.\frac{d}{d \epsilon}\right|_{\epsilon=0} a_{m-2}\left(e^{-2 \epsilon f} F, e^{-2 \epsilon f} D, \mathcal{B}\right)=0 \text { and } \\
& \left.\frac{d}{d \epsilon}\right|_{\epsilon=0} a_{n}\left(1, e^{-2 \epsilon F} D, \mathcal{B}\right)=(m-n) a_{n}(F, D, \mathcal{B}) .
\end{aligned}
$$

5. Let $P: C^{\infty}\left(V_{1}\right) \rightarrow C^{\infty}\left(V_{2}\right)$ be an elliptic complex of Dirac type. Let $D:=P^{*} P$ and let $\hat{D}:=P P^{*}$ be the associated operators of Laplace type. With suitable boundary conditions, we have:

$$
\begin{aligned}
& a_{m}(1, D, \mathcal{B})-a_{m}(1, \hat{D}, \hat{\mathcal{B}})=\operatorname{index}(P) \text { and } \\
& a_{n}(1, D, \mathcal{B})-a_{n}(1, \hat{D}, \hat{\mathcal{B}})=0 \text { if } n \neq m
\end{aligned}
$$

The invariants which do not involve the interaction between $V_{D}$ and $V_{N}$ were determined in [3, 13]. There are no interior invariants since 5 is odd. To complete the proof of Lemma 3.1, we must exhibit a spanning set for the space of boundary invariants which are homogeneous of order 4 ; assertion 2 and 3 of Lemma 3.2 show that the constants $w_{i}$ are dimension free. We can eliminate some terms from consideration:

Lemma 3.3 Let $X$ be an arbitrary scalar monomial.

1. The following invariants do not appear in $a_{5}: X \chi_{: a} E_{: a}, \chi_{: a} \chi_{: a b b}$, $\Omega_{a m}\left(S_{: a} \chi+\chi S_{: a}\right), \chi \chi_{: a} \chi_{: b} X_{a b}, \Omega_{a m} S_{: a}, F_{; m} \chi \chi_{: a} S_{: a},\left(\chi_{: a a} \chi-\chi \chi_{: a a}\right) E$, $X_{i j} \chi_{: a} \Omega_{k l}$, and $\chi_{: a} \Omega_{j k ; i}$. 
2. The following invariants are linear combinations of the invariants defin$\operatorname{ing} \mathcal{A}_{5}^{3}: \chi_{: a a} \chi_{: b b}, \chi_{: a b} \chi_{: a b}, \chi \chi_{: a a} \chi \chi_{: b b}, \chi \chi_{: a b} \chi_{: a b}, \chi_{: a b} \chi_{: a} \chi_{: b}$, $\chi_{: a b} \chi_{: b} \chi_{: a}$, and $\chi_{: a a} \chi_{: b} \chi_{: b}$.

3. We have the following relations: $\operatorname{Tr}\left(\chi_{: a} \chi_{: b} \chi_{: a} \chi_{: b}\right)=0$, $\operatorname{Tr}\left(\chi_{: a} X_{a}\right)=0,\left(\operatorname{Tr} \chi \chi_{: a} \chi_{: a} X\right)=0, \operatorname{Tr}\left(\chi_{: a b} X_{a b}\right)=0$, and $\operatorname{Tr}\left(\chi_{: a} X_{a}\right)=0$.

Proof: We use arguments of [2] to prove the first assertion. Let the smearing function $F$ be real. Suppose that the bundle $V$, the operator $D$, and the endomorphisms $S$ and $\chi$ are real. It then follows that $\operatorname{Tr}\left(F e^{-t D_{\mathcal{B}}}\right)$ is real. Thus all the coefficients must be real. Suppose next that $V$ is complex, that $\nabla$ is Hermitian, and that $E, S$, and $\chi$ are self-adjoint. Then the operator $D_{\mathcal{B}}$ is self-adjoint and again $\operatorname{Tr}\left(F e^{-t D_{\mathcal{B}}}\right)$ is real. The curvature tensor $\Omega_{i j}$ is anti-Hermitian as it is a commutator of two covariant derivatives. The terms in the first assertion have purely imaginary trace and thus do will not appear in the formula for $a_{5}^{\partial M}$.

Let $\{A, B\}:=A B+B A$ be the anti-commutator. We differentiate the identity $\chi^{2}=1$ to see

$$
\begin{aligned}
& \left\{\chi, \chi_{: a}\right\}=0, \quad\left\{\chi_{: a b}, \chi\right\}+\left\{\chi_{: a}, \chi_{: b}\right\}=0 \\
& \left\{\chi_{: a a b}, \chi\right\}+\left\{\chi_{: a a}, \chi_{: b}\right\}+2\left\{\chi_{: a b}, \chi_{: a}\right\}=0
\end{aligned}
$$

We use the Ricci identity to see that, at the cost of getting additional terms involving $\Omega$, the fourth derivative of $\chi$ can be reduced to terms with lower derivatives. Thus

$$
\left\{\chi_{: a a b b}, \chi\right\}+(\text { fewer derivatives in } \chi)=0 .
$$

We differentiate the relation $\chi S=S \chi=S$ to see that:

$$
\begin{aligned}
& S \chi_{: a}+S_{: a} \chi=S_{: a}=\chi_{: a} S+\chi S_{: a} \\
& \begin{aligned}
S_{: a b} & =S \chi_{: a b}+S_{: a} \chi_{: b}+S_{: b} \chi_{: a}+S_{: a b} \chi \\
& =\chi_{: a b} S+\chi_{: a} S_{: b}+\chi_{: b} S_{: a}+\chi S_{: a b} .
\end{aligned}
\end{aligned}
$$

We also observe that:

$$
\begin{aligned}
& \operatorname{Tr}\left(\chi_{: a} \chi_{: b} \chi_{: a b}\right)=\frac{1}{2} \operatorname{Tr}\left(\chi_{: a} \chi_{: b} \chi\left\{\chi, \chi_{: a b}\right\}\right)=\frac{1}{2} \operatorname{Tr}\left(\chi_{: a} \chi_{: a} \chi_{: b} \chi_{: b}\right) \\
& \operatorname{Tr}\left(\chi_{: a} \chi_{: a} \chi_{: b b}\right)=-\operatorname{Tr}\left(\chi_{: a} \chi_{: a} \chi_{: b} \chi_{: b}\right) .
\end{aligned}
$$

We use Equation (3) to see that we do not need a $\chi$, a $\chi_{: a}$ nor a $\chi_{\text {:aa }}$ touching an $S$. We use the identities $\operatorname{Tr}\left(\chi_{\chi_{a}} S_{: b} L_{a b}\right)=0$ and

$$
\operatorname{Tr}\left(S \chi_{: a b} L_{a b}\right)=-\operatorname{Tr}\left(S \chi_{: a} \chi_{: b} L_{a b}\right)=-\operatorname{Tr}\left(S_{: a} \chi_{: b} L_{a b}\right)
$$


together with Equation (3) to see that $\operatorname{Tr}\left(\chi_{: a} S\right)=0, \operatorname{Tr}\left(\chi S_{: b}\right)=\operatorname{Tr}\left(S_{: b}\right)$,

$$
\begin{aligned}
\operatorname{Tr}\left(\chi S_{: a} \chi S_{: a}\right) & =\operatorname{Tr}\left(2 \chi S_{: a} S_{: a}-S_{: a} S_{: a}\right), \text { and } \\
\operatorname{Tr}\left(\chi S_{: a b} L_{a b}\right) & =\operatorname{Tr}\left(S_{: a b} L_{a b}-S \chi_{: a b} L_{a b}-2 S_{: a} \chi_{: b} L_{a b}\right) \\
& =\operatorname{Tr}\left(S_{: a b} L_{a b}-S_{: a} \chi_{: b} L_{a b}\right) .
\end{aligned}
$$

In addition to the relations of Lemma 3.3, we note that terms obtained by commuting the order of derivatives are controlled by the Ricci identity.

Proof of Lemma 3.1: We have eliminated most of the additional possible terms which might otherwise be thought to appear in the local formula for $a_{5}$. Note that terms of the form $\chi_{: a b} X_{a b}$ are controlled by $\chi_{: a} \chi_{: b} X_{a b}$. There are, however, a few remaining terms to be eliminated. We order these terms by length and complete the proof by eliminating these terms.

1. (Terms of length 2). As $\Omega_{a b}$ is antisymmetric, we have $\operatorname{Tr}\left(\chi_{: a b} \Omega_{a b}\right)=0$.

2. (Terms of length 3). We use the Ricci identity to see that

$$
\chi \chi_{: a b} \Omega_{a b}=\frac{1}{2} \chi\left(\chi_{: a b}-\chi_{: b a}\right) \Omega_{a b}=\frac{1}{2} \chi\left[\chi, \Omega_{a b}\right] \Omega_{a b} .
$$

The terms on the right hand side are controlled and thus we may omit $\operatorname{Tr}\left(\chi \chi_{: a b} \Omega\right)$ from our list of invariants. A similar argument shows we may omit $\operatorname{Tr}\left(\chi_{: a b} \chi \Omega_{a b}\right)$ from our list of invariants. We use Lemma 3.3 to exclude the invariant $\chi_{: a a} E-\chi_{: a a} \chi E$. We control the invariant $\chi \chi_{: a a} E+\chi_{: a a} \chi E$ by $\chi_{: a} \chi_{: a} E$.

3. (Terms of length 4). By Equation (3), $\operatorname{Tr}\left(\chi \chi \chi_{a b} \chi \Omega_{a b}\right)=0$.

The following relations follow from Lemma 2.3 and from the product formula of assertion 2 of Lemma 3.2.

Lemma 3.4 We have $w_{3}+w_{4}=600, w_{19}=-180, w_{22}=-30, w_{5}+w_{7}=0$, and $w_{6}=120$.

\section{Two dimensional calculations}

Let $D$ be an operator of Laplace type on the trivial vector bundle over the cylinder $M:=S^{1} \times[0,1]$, which we endow with the standard flat metric. We specialize the formula for $a_{5}$ to the situation at hand. We set $L=0, R=0$, $F=1, m=2$, and $a=b=c=1$. This yields the expression:

$$
a_{5}(1, D, \mathcal{B})=5760^{-1}(4 \pi)^{-1 / 2} \operatorname{Tr}\left\{360 \chi E_{; 22}+1440 E_{; 2} S+720 \chi E^{2}\right.
$$




$$
\begin{aligned}
& +240 \chi E_{: 11}+960 S S_{: 11}+2880 E S^{2}+1440 S^{4}+w_{1} E^{2} \\
& +w_{2} \chi E \chi E+w_{3} S_{: 1} S_{: 1}+w_{4} \chi S_{: 1} S_{: 1}+w_{8} \Omega_{12} \Omega_{12} \\
& +w_{9} \chi \Omega_{12} \Omega_{12}+w_{10} \chi \Omega_{12} \chi \Omega_{12}+w_{11}\left(\Omega_{12} \chi S_{: 1}-\Omega_{12} S_{: 1} \chi\right) \\
& +w_{15} \chi \chi_{: 1} \Omega_{12 ; 2}+w_{18} \chi_{: 1} E_{: 1}+w_{19} \chi_{: 1} \chi_{: 1} E+w_{20} \chi_{: 1} \chi_{: 1} E \\
& +w_{21} \chi_{: 11} E+w_{32} \chi_{: 1} \chi_{: 1} \chi_{: 1} \chi_{: 1}+w_{33} \chi_{: 1} \chi_{: 1} \chi_{: 1} \chi_{: 1}+w_{34} \chi_{: 11} \chi_{: 11} \\
& \left.+w_{35} \chi_{: 11} \chi_{: 11}+w_{36} \chi_{: 1} \chi_{: 1} \chi_{: 11}+w_{37} \chi_{: 1} \chi_{: 111}\right\}[\partial M]
\end{aligned}
$$

We use the relation $w_{3}+w_{4}=600$ to see that

$$
\begin{aligned}
& \operatorname{Tr}\left(960 S S_{: 11}+w_{3} S_{: 1} S_{: 1}+w_{4} \chi S_{: 1} S_{: 1}\right)[\partial M] \\
= & \operatorname{Tr}\left(-360 S_{: 1} S_{: 1}-2 w_{4} \Pi_{-} S_{: 1} S_{: 1}\right)[\partial M]
\end{aligned}
$$

We integrate by parts to see $f_{: 1} g[\partial M]=-f g_{: 1}[\partial M]$. We use this relation to see $\operatorname{Tr}\left(\chi_{: 1} \chi_{: 1} \chi_{: 11}\right)[\partial M]=0$ and to simplify the expression for $a_{5}$. This shows:

$$
\begin{aligned}
a_{5}(1, & D, \mathcal{B})=5760^{-1}(4 \pi)^{-1 / 2} \operatorname{Tr}\left\{360 \chi E_{; 22}+1440 E_{; 2} S+720 \chi E^{2}\right. \\
& +2880 E S^{2}+1440 S^{4}+w_{1} E^{2}+w_{2} \chi E \chi E-360 S_{: 1} S_{: 1} \\
& -2 w_{4} \Pi_{-} S_{: 1} S_{: 1}+w_{8} \Omega_{12} \Omega_{12}+w_{9} \chi \Omega_{12} \Omega_{12}+w_{10} \chi \Omega_{12} \chi \Omega_{12} \\
& +w_{11}\left(\Omega_{12} \chi S_{: 1}-\Omega_{12} S_{: 1} \chi\right)+w_{15} \chi \chi_{: 1} \Omega_{12 ; 2}+w_{19} \chi_{: 1} \chi_{: 1} E \\
& +w_{20} \chi \chi_{: 1} \chi_{: 1} E+\left(240-w_{18}+w_{21}\right) \chi_{: 11} E \\
& \left.+\left(w_{32}+w_{33}\right) \chi_{: 1} \chi_{: 1} \chi_{: 1} \chi_{: 1}+\left(w_{34}+w_{35}-w_{37}\right) \chi_{: 11} \chi_{: 11}\right\}[\partial M] .
\end{aligned}
$$

Lemma 4.1 We have

\begin{tabular}{|l|l|l|l|}
\hline$w_{1}=-180$ & $w_{2}=180$ & $w_{3}=-120$ & $w_{4}=720$ \\
\hline$w_{8}=-45$ & $w_{9}=180$ & $w_{10}=-45$ & $w_{11}=360$ \\
\hline$w_{15}=90$ & $w_{19}=-180$ & $w_{20}=-90$ & $w_{21}-w_{18}=-60$ \\
\hline
\end{tabular}

Proof: We apply the local index formula of Lemma 3.2 (5). Let $i, j$, and $k$ be real skew-adjoint $4 \times 4$ matrices satisfying the quaternion relations. Let

$$
\begin{aligned}
& A:=a_{0}+i a_{1}+j a_{2}+k a_{3} \quad \text { for } \quad \vec{a} \in \mathbb{R} \oplus \sqrt{-1} \mathbb{R}^{3} \\
& B:=b_{0}+i b_{1}+j b_{2}+k b_{3} \quad \text { for } \quad \vec{b} \in \sqrt{-1} \mathbb{R} \oplus \mathbb{R}^{3}
\end{aligned}
$$

be matrix valued functions on $M$. Then $A^{*}=A$ and $B^{*}=-B$. Let

$$
P:=i \partial_{1}+j \partial_{2}+A+B \quad \text { and } \quad P^{*}:=i \partial_{1}+j \partial_{2}+A-B
$$

be operators of Dirac type and let $D:=P^{*} P$ and $\hat{D}:=P P^{*}$ be the associated operators of Laplace type. Let $\chi:=\sqrt{-1} i$; we note that $\chi^{2}=1, \chi i=i \chi$, and $\chi j=-j \chi$. Let $\Pi_{ \pm}:=\frac{1}{2}(1 \pm \chi)$ be the complementary projections on 
the \pm 1 eigenspaces of $\chi$. We define admissible boundary operators $\mathcal{B}$ and $\hat{\mathcal{B}}$ of mixed type which make $D$ and $\hat{D}$ self-adjoint:

$$
\mathcal{B} \phi:=\left.\left.\left(\Pi_{-} \phi\right)\right|_{\partial M} \oplus\left(\Pi_{-} P \phi\right)\right|_{\partial M}, \text { and } \hat{\mathcal{B}} \phi:=\left.\left.\left(\Pi_{-} \phi\right)\right|_{\partial M} \oplus\left(\Pi_{-} P^{*} \phi\right)\right|_{\partial M} .
$$

Since $P$ intertwines $D$ and $\hat{D}$ and since the index of this complex is zero,

$$
a_{n}(1, D, \mathcal{B})=a_{n}(1, \hat{D}, \hat{\mathcal{B}}) .
$$

If we interchange $B$ and $-B$, then we interchange the roles of $P$ and $P^{*}$ and the roles of $D$ and $\hat{D}$. Thus the terms of odd degree in $B$ must vanish in $a_{n}(1, D, \mathcal{B})$. Let $\dot{A}=\partial_{1} A, \dot{B}=\partial_{1} B, \tilde{A}=\partial_{2} A$, and $\tilde{B}=\partial_{2} B$. We first study the terms which are bilinear in the jets of $A$ and $B$. These terms change sign if we interchange the roles of $D$ and $\hat{D}$ and hence their coefficient in $a_{5}$ must be zero. Let $\mathcal{J}:=2 \operatorname{Tr}(I)$ and $\mathcal{K}:=2 \sqrt{-1} \operatorname{Tr}(I)$. We use Lemmas A.1-A.3 to obtain the following system of Equations.

$$
\begin{array}{lr}
0=2 w_{8}+2 w_{10}+2 w_{15} & \left(\tilde{a}_{0} \dot{b}_{3} \mathcal{J}\right) \\
0=-2 w_{8}+2 w_{10} & \left(\dot{a}_{0} \tilde{b}_{3} \mathcal{J}\right) \\
0=-2 w_{9}+2\left(240-w_{18}+w_{21}\right) & \left(\tilde{a}_{1} \dot{b}_{3} \mathcal{K}\right) \\
0=-2 w_{2}-2 w_{1} & \left(\tilde{a}_{3} \dot{b}_{0} \mathcal{J}\right) \\
0=-2 w_{2}+2 w_{1}+\frac{1}{2} 1440 & \left(\dot{a}_{3} \tilde{b}_{0} \mathcal{J}\right) \\
0=2\left(240-w_{18}+w_{21}\right)-360 & \left(\dot{a}_{3} \dot{b}_{2} \mathcal{K}\right) \\
0=4 w_{8}+4 w_{10}+1440-12 w_{15} & \left(\tilde{a}_{0} a_{0} b_{2} \mathcal{J}\right)
\end{array}
$$

This implies that:

$$
\begin{aligned}
& w_{8}=-45, w_{9}=180, w_{10}=-45, w_{2}=180, \\
& w_{1}=-180,\left(240-w_{18}+w_{21}\right)=180, w_{15}=90 .
\end{aligned}
$$

Next we set $a_{0}=a_{1}=a_{2}=0$, we let $a_{3}$ be a constant, and we assume $B=B\left(x_{1}\right)$. This yields the following system of Equations we use to complete the proof of Lemma 4.1 .

$$
\begin{aligned}
& 0=4 w_{20}-6\left(240-w_{18}+w_{21}\right)+2 \cdot 720 \quad\left(\dot{b}_{0} b_{3}^{2} \mathcal{K}, b_{0} \dot{b}_{3} b_{3} \mathcal{K}\right) \\
& 0=-8 w_{20}-2 w_{4}+8\left(240-w_{18}+w_{21}\right) \\
& +2(-360)-2880+2 \cdot 1440 \\
& 0=4 w_{8}+4 w_{10}-4 w_{15} \\
& -2\left(-4 w_{8}+4 w_{10}+4 w_{15}+4 w_{19}\right) \\
& 0=4 w_{19}+\frac{1}{2} 2880-2 w_{11} \text {. } \\
& \begin{array}{r}
\left(\dot{b}_{0} b_{3}^{2} \mathcal{K}, b_{0} \dot{b}_{3} b_{3} \mathcal{K}\right) \\
\left(a_{3} b_{2}^{3} \mathcal{K}\right) \\
\left(\dot{b}_{3} b_{3} b_{1} \mathcal{J}, \dot{b}_{1} b_{3}^{2} \mathcal{J}\right) \\
\left(\dot{b}_{1} b_{2}^{2} \mathcal{J}\right)
\end{array}
\end{aligned}
$$

\section{Calculations on the ball}

In this section, we compare the results of calculations on the ball performed in [5, 7] with the formula from Theorem 2.4 to establish the following result: 
Lemma 5.1 We have the following relations:

$$
\begin{aligned}
-135 & =2 w_{27}+4 w_{33}+2 w_{35} \\
705 & =16 w_{28}+16 w_{29}-4 w_{31}+16 w_{32}-16 w_{33}+16 w_{34}-16 w_{37} \\
1725 & =2 w_{3}+32 w_{26}-8 w_{30} \\
-675 & =32 w_{26} \\
1935 & =16 w_{28}+16 w_{29}-8 w_{30}+32 w_{32}+32 w_{34}+16 w_{35}+32 w_{36}-32 w_{37} \\
585 & =4 w_{27}-2 w_{31}+8 w_{32}+16 w_{33}+8 w_{34}+12 w_{35}-8 w_{36}-8 w_{37} .
\end{aligned}
$$

Proof: We start with the Dirac spinors. Let $\gamma_{i} \in M_{2^{[m / 2]}}$ be the Dirac gamma matrices; these satisfy the Clifford commutation relation $\gamma_{j} \gamma_{k}+\gamma_{k} \gamma_{j}=-2 \delta_{k j}$. Let $P=\gamma^{\mu} \nabla_{\mu}$ be an operator of Dirac type. We assume that the connection is compatible, in other words $\nabla \gamma=0$, or in index notation, $\left[\nabla_{\mu}, \gamma_{\nu}\right]=0$. For $m$ even, let

$$
\Gamma^{5}:=(\sqrt{-1})^{m / 2} \gamma_{1} \ldots \gamma_{m}
$$

We then have $\left(\Gamma^{5}\right)^{2}=1$ and $\Gamma^{5} \gamma_{j}+\gamma_{j} \Gamma^{5}=0$. We refer to [2] for further information concerning the spectral geometry of the Dirac operator with local boundary conditions. We use formulas from [2]. Consider the boundary value problem for the corresponding Laplacian $D=P^{2}$. We set

$$
\chi:=-\Gamma^{5} \gamma_{m} \text { and } S:=-\frac{1}{2} L_{a a} \Pi_{+}
$$

to define admissible boundary conditions. The endomorphism $E$, the Riemann curvature and $\Omega$ are zero. Since $L_{a b: c}=0$ and $L_{a b}=\delta_{a b}$, we have:

$$
\chi_{: a}=L_{a c} \Gamma^{5} \gamma_{c}, \chi_{: a b}=-L_{a c} L_{b c} \chi, S_{: a}=-\frac{1}{4} L_{b b} \chi_{: a}, S_{: a b}=\frac{1}{4} L_{d d} L_{a c} L_{b c} \chi
$$

Dowker, Apps, Kirsten and Bordag showed in [5] that:

$$
\begin{aligned}
a_{5}\left(1, D, \mathcal{B}_{S}\right)=\frac{2^{-m+1} 2^{m / 2} \sqrt{\pi}}{\Gamma\left(\frac{m}{2}\right)}( & -\frac{29(m-1)}{2560}+\frac{91(m-1)^{2}}{30720} \\
& \left.+\frac{11(m-1)^{3}}{3840}-\frac{89(m-1)^{4}}{122880}\right) .
\end{aligned}
$$

We have $\operatorname{Vol}\left(S^{m-1}\right)=2 \pi^{m / 2} / \Gamma\left(\frac{m}{2}\right)$. We compare coefficients of the powers of $m-1$ in the above equation with the general formula of Theorem 2.4 to establish the first three equations of Lemma 5.1.

Next we study vector fields (or 1-forms) on the unit ball with absolute boundary conditions. For these boundary conditions, $\chi$ can be viewed as a matrix acting in the tangent space to $M$ :

$$
\chi_{m m}=-1, \quad \chi_{b a}=\delta_{b a}, \text { and } \quad S=-\Pi_{+} .
$$


As above, $E=0, R=0$, and $\Omega=0$. We have that:

$$
\begin{aligned}
& \left(\chi_{: a}\right)_{m b}=\left(\chi_{: a}\right)_{b m}=-2 \delta_{a b} \\
& \left(\chi_{: a b}\right)_{m m}=4 \delta_{a b}, \quad\left(\chi_{: a b}\right)_{c d}=-2\left(\delta_{c d} \delta_{a d}+\delta_{a c} \delta_{b d}\right) .
\end{aligned}
$$

We use [7] (see also [8, 19]) to see the following relationship from which the final assertions of Lemma 5.1 follow:

$$
\begin{aligned}
\frac{(4 \pi)^{(m-1) / 2}}{\operatorname{Vol} S^{m-1}} a_{5}= & \frac{1309(m-1)}{15360}-\frac{6313(m-1)^{2}}{36864}+\frac{6359(m-1)^{3}}{46080} \\
& -\frac{26587(m-1)^{4}}{737280}+\frac{2041(m-1)^{5}}{737280} .
\end{aligned}
$$

\section{Conformal relations}

Lemma 6.1 We have the following relations:

\begin{tabular}{|l|l|l|l|}
\hline$w_{16}=120$ & $w_{18}=300$ & $w_{21}=240$ & $w_{23}=0$ \\
\hline$w_{24}=-60$ & $w_{25}=30$ & $w_{26}=-\frac{675}{32}$ & $w_{27}=-\frac{75}{4}$ \\
\hline$w_{28}=-\frac{195}{16}$ & $w_{29}=-\frac{675}{8}$ & $w_{30}=-330$ & $w_{31}=-300$ \\
\hline$w_{32}=\frac{15}{4}$ & $w_{33}=\frac{15}{8}$ & $w_{34}=-\frac{15}{4}$ & $w_{35}=-\frac{105}{2}$ \\
\hline$w_{36}=-15$ & $w_{37}=-\frac{135}{2}$ & $w_{38}=-210$ & $w_{39}=-\frac{165}{16}$ \\
\hline$w_{40}=-\frac{405}{8}$ & $w_{42}=-30$ & & \\
\hline
\end{tabular}

Proof: We use Lemma 3.2 (4). Consider the variations $D(\epsilon)=e^{-2 \epsilon f} D$, $g(\epsilon)=e^{2 \epsilon f} g$, and $F(\epsilon)=e^{-2 \epsilon f} F$. We set

$$
S(\epsilon)=e^{-\epsilon f} \Pi_{+}\left\{\omega_{m}(0)-\omega_{m}(\epsilon)+S\right\} .
$$

to fix the boundary conditions. Let $e_{i}$ be an orthonormal frame for the tangent and cotangent bundles of $M$ with respect to the reference metric $g(0)$. Let $e_{i}(\epsilon)=e^{-\epsilon f} e_{i}$ and $e^{i}(\epsilon)=e^{\epsilon f} e^{i}$ be the corresponding frames for the metric $g(\epsilon)$. We remark that contraction and differentiation do not commute;

$$
\left.\frac{d}{d \epsilon}\right|_{\epsilon=0}\left(\Phi_{i i}\right)=\left(\left.\frac{d}{d \epsilon}\right|_{\epsilon=0} \Phi\right)_{i i}-2 f \Phi_{i i}
$$

for example. Although the Christoffel symbols $\Gamma$ are not tensorial, their variation is tensorial. We have the following relations; a more extensive list is given in [3]. Conformal variations of the new invariants are listed in Appendix B.

$$
\begin{aligned}
& \left(\left.\frac{d}{d \epsilon}\right|_{\epsilon=0} \Gamma\right)_{i j}{ }^{k}=\delta_{i k} f_{; j}+\delta_{j k} f_{; i}-\delta_{i j} f_{; k}, \\
& \left(\left.\frac{d}{d \epsilon}\right|_{\epsilon=0} L\right)_{a b}=-\delta_{a b} f_{; m}-f L_{a b}, \\
& \left.\frac{d}{d \epsilon}\right|_{\epsilon=0}(S)=-f S+\Pi_{+} \frac{1}{2}(m-2) f_{; m}, \\
& \left.\frac{d}{d \epsilon}\right|_{\epsilon=0}(E)=-2 f E+\frac{1}{2}(m-2) f_{; i i}, \text { and } \\
& \left(\left.\frac{d}{d \epsilon}\right|_{\epsilon=0} R\right)_{i j k l}=\delta_{i k} f_{; j l}+\delta_{j l} f_{; i k}-\delta_{i \ell} f_{; j k}-\delta_{j k} f_{; i l}+2 f R_{i j k l} .
\end{aligned}
$$


The following relations are obtained from Lemma 3.2 (4) by comparing coefficients before various terms (listed on the left). Lemma 6.1 follows from these relations.

$$
\begin{aligned}
& \underline{\text { Term }} \quad \underline{\text { Coefficient }} \\
& F \chi_{: a} E_{: a} \quad 0=2 w_{18}-(m-3) w_{21}-360+1440(m-2) \\
& -240(m-3)-960(m-1)+240 \\
& F_{; m} \chi_{: a} S_{: a} \quad 0=(m-1) w_{30}-\frac{1}{2}(m-2) w_{3}+480(m-2) \\
& +w_{31}+(m-5) w_{38} \\
& F_{; m m} \chi_{: a} \chi_{: a} \quad 0=\frac{1}{2}(m-2) w_{19}-2(m-1) w_{22}-(m-1) w_{23} \\
& -w_{24}-w_{25}-(m-5) w_{42} \\
& F_{; m} L_{b b} \chi_{: a} \chi_{: a} \quad 0=\frac{1}{2}(m-2) w_{19}-2(m-1) w_{22}-w_{23}-w_{24}+w_{29} \\
& +2(m-1) w_{26}+2 w_{28}-\frac{1}{4}(m-2) w_{30} \\
& +(m-5) w_{39} \\
& F \chi_{: a a b} \chi: b \quad 0=(m-2) w_{19}-4(m-1) w_{22}-2 w_{23}-2(m-1) w_{24} \\
& -2 w_{25}-2(m-3) w_{34}+2 w_{35}+(m-1) w_{37} \\
& F \chi_{: a b} \chi_{: a b} \quad 0=(m-2) w_{19}-4(m-1) w_{22}-2 w_{23} \\
& -m w_{24}-w_{25}+4 w_{35} \\
& F_{; m} \chi_{: a} \chi_{: b} L_{a b} \quad 0=-\frac{1}{4}(m-2) w_{31}-(m-2) w_{24}-w_{25}+2 w_{27} \\
& +(m-1) w_{29}+(m-5) w_{40} \\
& F \chi_{: a a} \chi: b b \quad 0=-(m-2) w_{24}-w_{25}-2(m-3) w_{34}-2 w_{35} \\
& +(m-1) w_{37} \\
& F_{; m} \chi \chi_{: a} \Omega_{a m} \quad 0=+\frac{1}{2}(m-2) w_{11}-(m-1) w_{12}-2 w_{15}-w_{17} \\
& -(m-5) w_{41} \\
& F \chi_{: a} \chi_{: b} \Omega_{a b} \quad 0=w_{15}+(m-5) w_{16}+4(m-2) w_{19}-16(m-1) w_{22} \\
& -8 w_{23}-(2 m+4) w_{24}-2 w_{25}+12 w_{35} \\
& F \chi \chi_{: a} \Omega_{a b: b} \quad 0=-w_{15}-(m-5) w_{16}-2(m-2) w_{19}+8(m-1) w_{22} \\
& +4 w_{23}+4 w_{24}-4 w_{35} \\
& F \Omega_{a b} \Omega_{a b} \quad 0=-\frac{1}{2} w_{15}-\frac{1}{2}(m-5) w_{16}-(m-2) w_{24}-w_{25}+2 w_{35} \\
& f_{; m} F_{; m} \chi_{: a} \chi_{: a} \quad 0=w_{40}+5 w_{42}+6 w_{39}-\frac{5}{4} w_{38} \text {. }
\end{aligned}
$$

\section{Two more index theorem examples}

The only remaining universal constants to be determined are the coefficients of the following terms:

$$
\begin{aligned}
P:= & \frac{1}{2}\left(w_{5}-w_{7}\right) F\left(\Omega_{a b} \Omega_{a b}-\chi \Omega_{a b} \chi \Omega_{a b}\right)+w_{12} F \chi \chi_{: a} \Omega_{a m} L_{c c} \\
& +w_{13} F \chi_{: a} \chi_{: b} \Omega_{a b}+w_{14} F \chi \chi_{: a} \chi_{: b} \Omega_{a b} \\
& +w_{17} F \chi_{: a} \Omega_{b m} L_{a b}+w_{41} F_{; m} \chi \chi_{: a} \Omega_{a m} .
\end{aligned}
$$

Lemma 7.1 We have 


\begin{tabular}{|l|l|l|}
\hline$-\frac{1}{2}\left(w_{5}-w_{7}\right)=\frac{105}{4}$ & $w_{17}=-180$ & $w_{12}=-45$ \\
\hline$w_{13}=-180$ & $w_{14}=90$ & \\
\hline
\end{tabular}

Let $M$ be the generalized cylinder $M:=S^{1} \times S^{1} \ldots \times S^{1} \times[0,1]$ for $m$ even with the conformally flat metric

$$
d s^{2}=e^{2 f}\left(d x_{1}^{2}+\ldots+d x_{m}^{2}\right) .
$$

Let $\gamma_{i}$ be skew-adjoint matrices satisfying the Clifford relations. Let

$$
A:=e^{-f}\left(\gamma_{i} \partial_{i}\right) \text { and } A^{*}:=e^{-m f}\left(\gamma_{i} \partial_{i}\right) e^{(m-1) f} .
$$

Let $D^{[0]}:=A^{*} A$ and $D^{[1]}:=A A^{*}$ be the associated Laplacians. We set

$$
\begin{aligned}
& \Gamma^{5}:=(\sqrt{-1})^{m / 2} \gamma_{1} \ldots \gamma_{m}, \chi:=-\Gamma^{5} \gamma_{m} \\
& \mathcal{B}^{[0]} \phi:=\left.\left.\left(\Pi_{-} \phi\right)\right|_{\partial M} \oplus\left(\Pi_{-} A \phi\right)\right|_{\partial M}, \text { and } \\
& \mathcal{B}^{[1]} \phi:=\left.\left.\left(\Pi_{-} \phi\right)\right|_{\partial M} \oplus\left(\Pi_{-} A^{*} \phi\right)\right|_{\partial M} .
\end{aligned}
$$

We use the local index formula of Lemma 3.2 (5) to see that

$$
a_{5}\left(1, D^{[0]}, \mathcal{B}^{[0]}\right)=a_{5}\left(1, D^{[1]}, \mathcal{B}^{[1]}\right) .
$$

We use this relationship to derive certain additional relations among the coefficients appearing in Equation (田). Let a comma denote ordinary partial differentiation. For simplicity we put $\left.f\right|_{\partial M}=0$. This implies also $\left.f_{, a}\right|_{\partial M}=0$, but, in general, $\left.f_{, m a}\right|_{\partial M} \neq 0$. We compute:

$$
\begin{aligned}
D^{[1]}= & e^{-2 f}\left\{-\partial_{i}^{2}-\frac{1}{2} m f_{, i}\left[\gamma_{i}, \gamma_{j}\right] \partial_{j}+(2-m) f_{, i} \partial_{i}\right. \\
& \left.\quad+(m-1) f_{, i} f_{, i}-(m-1) f_{, i i}\right\}, \\
D^{[0]}= & e^{-2 f}\left\{-\partial_{i}^{2}+\frac{1}{2}(m-2) f_{, i}\left[\gamma_{i}, \gamma_{j}\right] \partial_{j}+(2-m) f_{, i} \partial_{i}\right\}, \\
\omega_{i}^{[1]}= & -\frac{1}{4} m\left[\gamma_{i}, \gamma_{j}\right] f_{, j}, \\
\Omega_{i j}^{[1]}= & -\frac{1}{4} m\left\{f_{, k i}\left[\gamma_{j}, \gamma_{k}\right]-f_{, k j}\left[\gamma_{i}, \gamma_{k}\right\}\right. \\
& +\frac{1}{4} m^{2}\left\{-f_{, j} f_{, k}\left[\gamma_{i}, \gamma_{k}\right]+f_{, i} f_{, k}\left[\gamma_{j}, \gamma_{k}\right]+f_{, k} f_{, k}\left[\gamma_{i}, \gamma_{j}\right]\right\}, \\
E^{[1]}= & e^{-2 f}(m-1)\left\{f_{, i i}+\frac{1}{4} f_{, i} f_{, i}\left(m^{2}-4\right)\right\}, \\
S^{[1]}= & (m-1) f_{, m} \Pi_{+}, \\
\omega_{i}^{[0]}= & \frac{1}{4}(m-2)\left[\gamma_{i}, \gamma_{j}\right] f_{, j}, \\
\Omega_{i j}^{[0]}= & \frac{1}{4}(m-2)\left\{f_{, k i}\left[\gamma_{j}, \gamma_{k}\right]-f_{, k j}\left[\gamma_{i}, \gamma_{k}\right\}\right. \\
& +\frac{1}{4}(m-2)^{2}\left\{-f_{, j} f_{, k}\left[\gamma_{i}, \gamma_{k}\right]+f_{, i} f_{, k}\left[\gamma_{j}, \gamma_{k}\right]+f_{, k} f_{, k}\left[\gamma_{i}, \gamma_{j}\right]\right\}, \\
E^{[0]}= & \frac{1}{4} e^{-2 f}(m-1)(m-2)^{2} f_{, i} f_{, i}, \text { and } \\
S^{[0]}= & 0 .
\end{aligned}
$$


The metric and hence the Riemann tensor is the same for both operators:

$$
\begin{gathered}
R_{j k l}^{i}=f_{, j} f_{, k} \delta_{i l}+f_{, i} f_{, l} \delta_{j k}-f_{, j} f_{, l} \delta_{i k}-f_{, i} f_{, k} \delta_{j l} \\
+f_{, p} f_{, p}\left(\delta_{j l} \delta_{i k}-\delta_{j k} \delta_{i l}\right) .
\end{gathered}
$$

We can now compute the invariants $a_{5}\left(1, D^{[i]}, \mathcal{B}^{[i]}\right)$. In Equation (47), the terms $\frac{1}{2}\left(w_{5}-w_{7}\right), w_{12}, w_{13}$ and $w_{17}$ contribute to $a_{5}$ in this setting. The invariants that appear in these contributions are $f_{, a m} f_{, a m}, f_{, m m} f_{, m}^{2}, f_{, m}^{4}$, and thus only terms of these types need to be kept during the calculation. In Appendix $\mathrm{C}$ we have listed all contributions appearing in $a_{5}\left(1, D^{[1]}, \mathcal{B}^{[1]}\right)-$ $a_{5}\left(1, D^{[0]}, \mathcal{B}^{[0]}\right)$. We therefore have the relations:

$$
\begin{aligned}
& \text { Term } \quad \underline{\text { Coefficient }} \\
& f_{, a m} f_{, a m} \quad 0=105+4(1 / 2)\left(w_{5}-w_{7}\right) \\
& f_{, m}^{2} f_{, m m} \quad 0=135-w_{12}+w_{17}+m\left(45+w_{12}\right) \\
& f_{, m}^{4} \quad 0=180+w_{13} \text {. }
\end{aligned}
$$

We use Lemma 3.4 to determine $w_{5}$ and $w_{7} ; w_{41}$ is now determined by the conformal relation of the previous section. This determines all the coefficients but $w_{14}$.

Let $M$ be as in Lemma 6.1, let $A=\gamma_{i}\left(\partial_{i}+\chi f_{i}\right)$, let $f_{m}$ be imaginary, and $f_{a}$ be real. It is obvious that $a_{5}\left(1, A^{*} A\right)=a_{5}\left(1, A A^{*}\right)$. Since $A^{*}=\gamma_{i}\left(\partial_{i}-\chi f_{i}\right)$, the heat kernel for $A A^{*}$ is obtained from that for $A^{*} A$ by changing sign before $f_{i}$. Therefore, all coefficients before odd powers of $f_{i}$ must vanish. Thus the coefficient before $f_{m}^{2} f_{a, a}$ in $a_{5}\left(1, A^{*} A\right)$ is zero. As above, $S=0$. We compute:

$$
\begin{aligned}
& \omega_{a}= \frac{1}{2}\left[\gamma_{b}, \gamma_{a}\right] \chi f_{b}-\gamma_{a} \gamma_{m} \chi f_{m} \\
& \omega_{m}= \chi f_{m} \\
& \Omega_{a m}= f_{m, a} \chi-2 \gamma_{a} \gamma_{m} f_{m}^{2} \\
& \Omega_{a b}=- \frac{1}{2}\left(f_{c, a}\left[\gamma_{b}, \gamma_{c}\right]-f_{c, b}\left[\gamma_{a}, \gamma_{c}\right]\right) \chi-\left(f_{m, b} \gamma_{a}-f_{m, a} \gamma_{b}\right) \gamma_{m} \chi \\
& \quad-\left[\gamma_{a}, \gamma_{b}\right] f_{m}^{2} \\
& \quad+\frac{1}{2} f_{c} f_{m}\left(-2 \gamma_{a} \gamma_{c} \gamma_{b}+2 \gamma_{b} \gamma_{c} \gamma_{a}+\gamma_{c}\left[\gamma_{a}, \gamma_{b}\right]+\left[\gamma_{a}, \gamma_{b}\right] \gamma_{c}\right) \\
& E=\chi f_{a, a}-(m-1) f_{m}^{2}+2(m-3) \gamma_{a} \gamma_{m} f_{a} f_{m} .
\end{aligned}
$$

Since we are looking for the terms with $f_{m}^{2} f_{a, a}$, all derivatives with respect to the $m$-th coordinate are not considered. We compute:

$$
\begin{aligned}
& \chi_{: a}=-2 f_{m} \gamma_{a} \gamma_{m} \\
& \chi_{: a b}=-2 \gamma_{a} \gamma_{m} f_{m, b}+\left(\left[\gamma_{c}, \gamma_{b}\right] \gamma_{a}+\gamma_{a}\left[\gamma_{c}, \gamma_{b}\right]\right) \gamma_{m} \chi f_{c} f_{m}+4 \delta_{a b} \chi f_{m}^{2} \\
& \chi_{: a a}=-2 \gamma_{a} \gamma_{m} f_{m, a}+4(m-1) f_{m}^{2} \chi .
\end{aligned}
$$


Only four invariants contain $f_{m}^{2} f_{a, a}$ :

\begin{tabular}{|l|l|l|}
\hline Invariant & Coefficient of $f_{m}^{2} f_{a, a}$ & Coefficient in $a_{5}$ \\
\hline$\chi \chi_{: a} \chi_{: b} \Omega_{a b}$ & $8(m-2)$ & $w_{14}$ \\
$\chi E^{2}$ & $-2(m-1)$ & 720 \\
$\chi_{: a} E_{: a}$ & $-2(m+1)$ & -180 \\
$\chi \chi_{: a} \chi_{: a} E$ & $-4(m-1)$ & -90 \\
\hline
\end{tabular}

Since all coefficients before odd powers of $f_{i}$ must vanish, $w_{14}=90$. This completes the proof of Lemma 7.1 and thereby of the main result of this paper, Theorem 2.4.

\section{A Appendix}

We adopt the notation of $\S$. Let

$$
\begin{array}{ll}
M=S^{1} \times[0,1], & \chi:=\sqrt{-1} i, \\
A:=a_{0}+i a_{1}+j a_{2}+k a_{3}, & B:=b_{0}+i b_{1}+j b_{2}+k b_{3}, \\
P:=i \partial_{1}+j \partial_{2}+A+B, & P^{*}:=i \partial_{1}+j \partial_{2}+A-B, \\
D=P^{*} P, &
\end{array}
$$

A section $f$ satisfies the boundary conditions $(\mathcal{B} f=0)$ if and only if we have $\left.\Pi_{-} f\right|_{\partial M}=0$ and $\left.\Pi_{-} P f\right|_{\partial M}=0$.

Lemma A.1 We have the following relations:

$$
\begin{aligned}
& \omega_{1}=a_{1}-a_{0} i+b_{3} j-b_{2} k \text { and } \omega_{2}=a_{2}-a_{0} j-b_{3} i+b_{1} k \text {. } \\
& \Omega_{12}=\left(\dot{a}_{2}-\tilde{a}_{1}\right)+\left(-\dot{b}_{3}+\tilde{a}_{0}+2 b_{3} b_{1}-2 a_{0} b_{2}\right) i+\left(-\dot{a}_{0}-\tilde{b}_{3}+2 a_{0} b_{1}+2 b_{2} b_{3}\right) j \\
& +\left(\dot{b}_{1}+\tilde{b}_{2}+2 a_{0}^{2}+2 b_{3}^{2}\right) k . \\
& E=\left(\dot{b}_{1}+\tilde{b}_{2}+a_{0}^{2}+a_{3}^{2}+b_{0}^{2}+b_{3}^{2}\right)+\left(-\dot{b}_{0}-\tilde{a}_{3}+2 a_{3} b_{2}+2 b_{0} b_{1}\right) i \\
& +\left(\dot{a}_{3}-\tilde{b}_{0}-2 a_{3} b_{1}+2 b_{0} b_{2}\right) j+\left(-\dot{a}_{2}+\tilde{a}_{1}-2 a_{0} a_{3}+2 b_{0} b_{3}\right) k . \\
& \chi_{: 1}=\sqrt{-1}\left(-2 b_{3} k-2 b_{2} j\right), \chi \chi_{: 1}=2\left(b_{2} k-b_{3} j\right) \text { and } \chi_{: 1}^{2}=4 b_{3}^{2}+4 b_{2}^{2} \text {. } \\
& \chi_{: 11}=\sqrt{-1}\left(\left(-4 b_{3}^{2}-4 b_{2}^{2}\right) i+\left(-2 \dot{b}_{2}-4 a_{0} b_{3}\right) j+\left(-2 \dot{b}_{3}+4 a_{0} b_{2}\right) k\right) \text {. } \\
& S=\Pi_{+}\left(\sqrt{-1} a_{3}+b_{2}\right) \text {. } \\
& S_{: 1}=\Pi_{+}\left(\sqrt{-1} \dot{a}_{3}+\dot{b}_{2}\right)+\sqrt{-1}\left(\sqrt{-1} a_{3}+b_{2}\right)\left(-b_{3} k-b_{2} j\right) \text {. } \\
& \chi_{: 1} S-S \chi_{: 1}=2\left(\sqrt{-1} a_{3}+b_{2}\right)\left(b_{3} j-b_{2} k\right) \text {. }
\end{aligned}
$$

Proof: We compute that:

$$
\begin{aligned}
& D=P^{*} P=-\partial_{1}^{2}-\partial_{2}^{2}+(i A+A i+i B-B i) \partial_{1}+(j A+A j+j B-B j) \partial_{2} \\
& \quad+i \dot{A}+i \dot{B}+j \tilde{A}+j \tilde{B}+A B-B A+A^{2}-B^{2} \\
& \omega_{1}=-\frac{1}{2}(i A+A i+i B-B i)=a_{1}-a_{0} i+b_{3} j-b_{2} k \\
& \omega_{2}=-\frac{1}{2}(j A+A j+j B-B j)=a_{2}-a_{0} j-b_{3} i+b_{1} k \\
& \omega_{1} \omega_{2}-\omega_{2} \omega_{1}=2 a_{0}^{2} k+2 a_{0} b_{1} j+2 b_{3}^{2} k+2 b_{3} b_{1} i-2 a_{0} b_{2} i+2 b_{2} b_{3} j \\
& \Omega_{12}=\partial_{1} \omega_{2}-\partial_{2} \omega_{1}+\omega_{1} \omega_{2}-\omega_{2} \omega_{1} \\
& \quad=\left(\dot{a}_{2}-\tilde{a}_{1}\right)+\left(-\dot{b}_{3}+\tilde{a}_{0}+2 b_{3} b_{1}-2 a_{0} b_{2}\right) i+\left(-\dot{a}_{0}-\tilde{b}_{3}+2 a_{0} b_{1}+2 b_{2} b_{3}\right) j
\end{aligned}
$$




$$
\begin{aligned}
& +\left(\dot{b}_{1}+\tilde{b}_{2}+2 a_{0}^{2}+2 b_{3}^{2}\right) k \\
E= & \mathcal{E}_{1}+\mathcal{E}_{2} \text { for } \\
\mathcal{E}_{1}= & -i \dot{A}-i \dot{B}-j \tilde{A}-j \tilde{B}-\dot{\omega}_{1}-\tilde{\omega}_{2} \\
= & -i \dot{A}-i \dot{B}+\frac{1}{2}(i \dot{A}+\dot{A} i+i \dot{B}-\dot{B} i)-j \tilde{A}-j \tilde{B}+\frac{1}{2}(j \tilde{A}+\tilde{A} j+j \tilde{B}-\tilde{B} j) \\
= & \frac{1}{2}(\dot{A} i-i \dot{A}-i \dot{B}-\dot{B} i)+\frac{1}{2}(\tilde{A} j-j \tilde{A}-j \tilde{B}-\tilde{B} j) \\
= & \dot{a}_{3} j-\dot{a}_{2} k+\dot{b}_{1}-\dot{b}_{0} i-\tilde{a}_{3} i+\tilde{a}_{1} k+\tilde{b}_{2}-\tilde{b}_{0} j \\
\mathcal{E}_{2}= & B^{2}-A^{2}+B A-A B-\omega_{1}^{2}-\omega_{2}^{2} \\
= & b_{0}^{2}-b_{1}^{2}-b_{2}^{2}-b_{3}^{2}+2 b_{0} b_{1} i+2 b_{0} b_{2} j+2 b_{0} b_{3} k \\
& -a_{0}^{2}+a_{1}^{2}+a_{2}^{2}+a_{3}^{2}-2 a_{0} a_{1} i-2 a_{0} a_{2} j-2 a_{0} a_{3} k \\
& +2\left(a_{2} b_{1}-a_{1} b_{2}\right) k+2\left(a_{3} b_{2}-a_{2} b_{3}\right) i+2\left(a_{1} b_{3}-a_{3} b_{1}\right) j \\
& -a_{1}^{2}+a_{0}^{2}+b_{3}^{2}+b_{2}^{2}+2 a_{1} a_{0} i-2 a_{1} b_{3} j+2 a_{1} b_{2} k \\
& -a_{2}^{2}+a_{0}^{2}+b_{3}^{2}+b_{1}^{2}+2 a_{2} a_{0} j+2 a_{2} b_{3} i-2 a_{2} b_{1} k \\
= & a_{0}^{2}+a_{3}^{2}+b_{0}^{2}+b_{3}^{2}-2 a_{0} a_{3} k+2 a_{3} b_{2} i-2 a_{3} b_{1} j \\
& +2 b_{0} b_{1} i+2 b_{0} b_{2} j+2 b_{0} b_{3} k \\
\chi_{: 1}= & \left.\sqrt{-1}\left[\omega_{1}, i\right)\right]=\sqrt{-1}\left[-a_{0} i+b_{3} j-b_{2} k, i\right] \\
= & 2 \sqrt{-1}\left(-b_{3} k-b_{2} j\right) \\
\chi_{: 11}= & \partial_{1} \chi_{: 1}+\left[\omega_{1}, \chi_{: 1}\right] \\
= & \sqrt{-1}\left[-2 b_{3} k-2 \dot{b}_{2} j-4 a_{0} b_{3} j+4 a_{0} b_{2} k-4 b_{3}^{2} i-4 b_{2}^{2} i\right] .
\end{aligned}
$$

Suppose $\Pi_{-} f=0$ on the boundary. Then

$$
\begin{aligned}
& \Pi_{-} P f=\Pi_{-}\left(i \partial_{1}+j \partial_{2}-j(j A+j B)\right) f \\
& =j \Pi_{+}\left(\partial_{2}+\omega_{2}-\omega_{2}-j A-j B\right) \Pi_{+} f \\
& S=\Pi_{+}\left(\frac{1}{2}(j A+A j+j B-B j)-j A-j B\right) \Pi_{+} \\
& =\frac{1}{2} \Pi_{+}(-j A+A j-j B-B j) \Pi_{+} \\
& =\Pi_{+}\left(-a_{3} i+a_{1} k+b_{2}-b_{0} j\right) \Pi_{+} \\
& =\Pi_{+}\left(-i a_{3}+b_{2}\right)=\Pi_{+}\left(\sqrt{-1} a_{3}+b_{2}\right) \\
& S_{: 1}=\Pi_{+}\left(\sqrt{-1} \dot{a}_{3}+\dot{b}_{2}\right)+\frac{1}{2} \chi_{: 1}\left(\sqrt{-1} a_{3}+b_{2}\right) .
\end{aligned}
$$

The Lemma now follows.

If $\mathcal{E}$ is a local invariant, let $\mu(\mathcal{E})=\operatorname{Tr}(\mathcal{E}(D, \mathcal{B}))-\operatorname{Tr}(\mathcal{E}(\hat{D}, \hat{B}))$. Then $\mu\left(a_{n}(1, \cdot)\right)[\partial M]=0$. We compute $\mu(\mathcal{E})$ for the terms appearing in the formula for $a_{5}$ contained in $\S$. We organize these terms into two lemmas to group the data involved. In Lemma A.2, we determine the terms which are bilinear in $A$ and $B$ or which involve $\tilde{a}_{0} a_{0} b_{2}$. In Lemma A.3, we study terms in $a_{3}$ and in the jets of $B$.

Lemma A.2 Terms which are bilinear in $A$ and $B$, and the term $a_{0} b_{2} \tilde{a}_{0} \mathcal{J}$.

$$
\begin{aligned}
& \mu\left(\Omega_{12}^{2}\right)=2\left(\tilde{a}_{0} \dot{b}_{3}-\dot{a}_{0} \tilde{b}_{3}\right) \mathcal{J}+4 a_{0} b_{2} \tilde{a}_{0} \mathcal{J}+\ldots \\
& \mu\left(\chi \Omega_{12}^{2}\right)=2\left\{\dot{a}_{2} \dot{b}_{3}-\tilde{a}_{1} \dot{b}_{3}\right\} \mathcal{K}+\ldots \\
& \mu\left(\chi \Omega_{12} \chi \Omega_{12}\right)=\left(2 \tilde{a}_{0} \dot{b}_{3}+2 \dot{a}_{0} \tilde{b}_{3}+4 a_{0} b_{2} \tilde{a}_{0}\right) \mathcal{J}+\ldots \\
& \mu\left(\chi E^{2}\right)=\left(2 \tilde{a}_{3} \dot{b}_{1}+2 \tilde{a}_{3} \tilde{b}_{2}\right) \mathcal{K}+\ldots \\
& \mu(\chi E \chi E)=-2\left(\tilde{a}_{3} \dot{b}_{0}+\dot{a}_{3} \tilde{b}_{0}\right) \mathcal{J}+\ldots \\
& \mu\left(E^{2}\right)=2\left(-\tilde{a}_{3} \dot{b}_{0}+\dot{a}_{3} \tilde{b}_{0}\right) \mathcal{J}+\ldots
\end{aligned}
$$




$$
\begin{aligned}
& \mu\left(\chi_{: 11} E\right)=2\left(\dot{a}_{3} \dot{b}_{2}-\dot{a}_{2} \dot{b}_{3}+\tilde{a}_{1} \dot{b}_{3}\right) \mathcal{K}+\ldots \quad\left(240-w_{18}+w_{21}\right) \\
& \mu\left(\chi_{: 11} \chi_{: 11}\right)=0+\ldots \quad\left(w_{34}+w_{35}-w_{37}\right) \\
& \mu\left(\chi E_{: 22}\right)=2\left(-\partial_{2}^{2}\left(a_{3} b_{2}\right)+2 b_{1} \partial_{2} \partial_{1} a_{3}+\dot{a}_{3} \tilde{b}_{1}\right) \mathcal{K}+\ldots \\
& \mu\left\{S_{: 1} S_{: 1}\right\}=\dot{a}_{3} \dot{b}_{2} \mathcal{K}+\ldots \\
& \mu\left(S E_{: 2}\right)=\frac{1}{2}\left(a_{3} \partial_{1} \partial_{2} b_{1}+a_{3} \partial_{2}^{2} b_{2}+b_{2} \partial^{2} a_{3}\right) \mathcal{K} \\
& \left(-\frac{1}{2} a_{3} \partial_{1} \partial_{2} b_{0}+\tilde{a}_{0} a_{0} b_{2}\right) \mathcal{J}+\ldots \\
& \mu\left(S^{2} E\right)=0+\ldots \\
& \mu\left(S^{4}\right)=0+\ldots \\
& \mu\left(\Pi_{-} S_{: 1} S_{: 1}\right)=0+\ldots \\
& \mu\left(\chi_{: 1} \chi_{: 1} E\right)=0+\ldots \\
& \mu\left(\chi \chi_{: 1} \chi_{: 1} E\right)=0+\ldots \\
& \mu\left(\chi_{: 1}^{4}\right)=0+\ldots \\
& \mu\left(\left(S \chi_{: 1}-\chi_{: 1} S\right) \Omega_{12}\right)=0+\ldots \\
& \mu\left(\left(\chi \chi_{: 1} \Omega_{12: 2}\right)\right)=-2 b_{3} \partial_{2} \partial_{1} a_{0} \mathcal{J}-12 a_{0} b_{2} \tilde{a}_{0} \mathcal{J}+\ldots
\end{aligned}
$$

Lemma A.3 $a_{0}=a_{1}=a_{2}=0$, let $a_{3}$ be constant, and let $B=B\left(x_{1}\right)$.

$$
\begin{aligned}
& \mu\left(\Omega_{12}^{2}\right)=\left(4 \dot{b}_{3} b_{3} b_{1}-4 \dot{b}_{1} b_{3}^{2}\right) \mathcal{J} \\
& \mu\left(\chi \Omega_{12}^{2}\right)=0 \text {. } \\
& \mu\left(\left(\chi \Omega_{12}\right)^{2}\right)=\left(4 \dot{b}_{3} b_{3} b_{1}+4 \dot{b}_{1} b_{3}^{2}\right) \mathcal{J} \\
& \mu\left((\chi E)^{2}\right)=\left\{2 \dot{b}_{1}\left(a_{3}^{2}+b_{0}^{2}+b_{3}^{2}\right)-4\left(-\dot{b}_{0}+2 a_{3} b_{2}\right) b_{0} b_{1}\right. \\
& \left.-8 a_{3} b_{1} b_{0} b_{2}\right\} \mathcal{J} \\
& \mu\left(E^{2}\right)=\left\{2 \dot{b}_{1}\left(a_{3}^{2}+b_{0}^{2}+b_{3}^{2}\right)+4 \dot{b}_{0} b_{0} b_{1}\right\} \mathcal{J} \\
& \mu\left(\chi E^{2}\right)=\left\{-2\left(a_{3}^{2}+b_{0}^{2}+b_{3}^{2}\right)\left(-\dot{b}_{0}+2 a_{3} b_{2}\right)\right. \\
& \left.-4 \dot{b}_{1} b_{0} b_{1}\right\} \mathcal{K} \\
& \mu\left(\chi_{: 11} E\right)=\left\{4\left(-\dot{b}_{0}+2 a_{3} b_{2}\right)\left(b_{3}^{2}+b_{2}^{2}\right)+4 b_{0} b_{2} \dot{b}_{2}\right. \\
& \left.+4 b_{0} b_{3} \dot{b}_{3}\right\} \mathcal{K} \\
& \mu\left(\chi_{: 11} \chi_{: 11}\right)=0 \\
& \mu\left(\chi E_{: 22}\right)=\left(-4 b_{1}^{2} \dot{b}_{0}+8 b_{1}^{2} a_{3} b_{2}\right) \mathcal{K} \\
& \mu\left\{S_{: 1} S_{: 1}\right\}=\left\{2 a_{3} b_{2}\left(b_{3}^{2}+b_{2}^{2}\right)\right\} \mathcal{K} \\
& \mu\left(S E_{: 2}\right)=\left\{-2 a_{3} b_{1}^{2} b_{2}\right\} \mathcal{K}-\left\{2 a_{3} b_{0} b_{1} b_{2}\right\} \mathcal{J} \\
& \mu\left(S^{2} E\right)=\left\{\frac{1}{2}\left(b_{2}^{2}-a_{3}^{2}\right) \dot{b}_{1}+2 a_{3} b_{0} b_{1} b_{2}\right\} \mathcal{J} \\
& \left\{-\frac{1}{2}\left(b_{2}^{2}-a_{3}^{2}\right)\left(-\dot{b}_{0}+2 a_{3} b_{2}\right)+a_{3} b_{2}\left(a_{3}^{2}+b_{0}^{2}+b_{3}^{2}\right)\right\} \mathcal{K} \\
& \mu\left(S^{4}\right)=\left(2 a_{3} b_{2}^{3}-2 a_{3}^{3} b_{2}\right) \mathcal{K} \\
& \mu\left(\Pi_{-} S_{: 1} S_{: 1}\right)=a_{3} b_{2}\left(b_{3}^{2}+b_{2}^{2}\right) \mathcal{K} \\
& \mu\left(\chi_{: 1} \chi_{: 1} E\right)=\left(4 b_{3}^{2}+4 b_{2}^{2}\right) \dot{b}_{1} \mathcal{J} \\
& \mu\left(\chi \chi_{: 1} \chi_{: 1} E\right)=-4\left(b_{3}^{2}+b_{2}^{2}\right)\left(-\dot{b}_{0}+2 a_{3} b_{2}\right) \mathcal{K} \\
& \mu\left(\chi_{: 1}^{4}\right)=0 \text {. } \\
& \mu\left(\left(S \chi_{: 1}-\chi_{: 1} S\right) \Omega_{12}\right)=-2 b_{2}^{2} \dot{b}_{1} \mathcal{J} \\
& \mu\left(\chi \chi_{: 1} \Omega_{12: 2}\right)=-4 \dot{b}_{3} b_{3} b_{1}+4 \dot{b}_{1} b_{3}^{2} \text {. } \\
& \left(w_{9}=180\right) \\
& \left(w_{10}\right) \\
& \left(240-w_{18}+w_{21}\right) \\
& \left(w_{34}+w_{35}-w_{37}\right) \\
& \left(w_{20}\right) \\
& \left(w_{32}+w_{33}\right) \\
& \left(w_{15}\right)
\end{aligned}
$$




\section{B Appendix}

Let $d s^{2}(\epsilon)=e^{2 \epsilon F} d s^{2}$ define a conformal variation. In this appendix, we summarize the computation of the conformal variations we needed in Section 6. We integrate by parts where necessary to bring the variations into standard form so that $F$ is not differentiated with respect to tangential coordinates. We will be dealing with terms which are homogeneous of order 4 . If $F$ is constant, then $\left.\frac{d}{d \epsilon}\right|_{\epsilon=0} X=-4 F X$. To avoid writing the conformal weight term repeatedly, we define $\mathcal{L} X:=\left.\frac{d}{d \epsilon} X\right|_{\epsilon=0}+4 F X$. Note that $\mathcal{L} X=0$ if $X$ only involves $\chi$ and $\Omega$ terms; consequently these terms have been omitted in the interests of brevity.

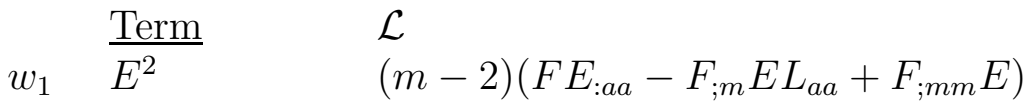

$$
\begin{aligned}
& w_{2} \quad \chi E \chi E \quad(m-2)\left(F E_{: a a}-F_{; m} E L_{a a}+F_{; m m} E\right) \\
& w_{3} \quad S_{: a} S_{: a} \quad 2 F S_{: a} S_{: a}+2 F S S_{: a a}-(m-2) F_{; m} S_{: a a} \\
& +\frac{m-2}{2} F_{; m} \chi_{: a} S_{: a} \\
& w_{4} \quad \chi S_{: a} S_{: a} \quad 2 F S_{: a} S_{: a}+2 F S S_{: a a}-(m-2) F_{; m} S_{: a a} \\
& w_{11} \quad \Omega_{a m}\left[\chi, S_{: a}\right] \quad \frac{m-2}{2} F_{; m} \chi \chi_{: a} \Omega_{a m} \\
& w_{12} \quad \chi \chi_{: a} \Omega_{a m} L_{c c} \quad-(m-1) F_{; m} \chi \chi_{: a} \Omega_{a m} \\
& w_{15} \quad \chi \chi_{: a} \Omega_{a m ; m} \quad F \chi_{: a} \chi_{: b} \Omega_{a b}-F \chi \chi_{: a} \Omega_{a b: b}-\frac{1}{2} F \Omega_{a b} \Omega_{a b} \\
& +\frac{1}{2} F \chi \Omega_{a b} \chi \Omega_{a b}-2 \chi \chi: a \Omega_{a m} F_{; m} \\
& w_{16} \quad \chi \chi_{: a} \Omega_{a b: b} \quad(m-5) F \chi_{: a} \chi_{: b} \Omega_{a b}-(m-5) \chi_{: a} \Omega_{a b: b} \\
& +\frac{m-5}{2} F\left(-\Omega_{a b} \Omega_{a b}+\chi \Omega_{a b} \chi \Omega_{a b}\right) \\
& w_{17} \quad \chi \chi_{: a} \Omega_{b m} L_{a b} \quad-\chi \chi_{: a} \Omega_{a m} F_{; m} \\
& w_{18} \quad \chi_{: a} E_{: a} \quad 2 F \chi_{: a a} E+2 F \chi_{: a} E_{: a} \\
& w_{19} \quad \chi_{: a} \chi_{: a} E \quad \frac{m-2}{2} \chi_{: a} \chi_{: a} F_{; m m}-\frac{m-2}{2} \chi_{: a} \chi_{: a} L_{c c} F_{; m} \\
& +(m-2) \chi_{: a b} \chi_{: a b} F+(m-2) \chi_{: b b a} \chi_{: a} F \\
& +4(m-2) \chi_{: a} \chi_{: b} \Omega_{a b} F-2(m-2) \chi_{: a} \Omega_{a b: b} F \\
& -(m-2) \chi_{: a} \chi_{: b} F\left(-\rho_{a b}+R_{m b a m}-L_{a b} L_{c c}+L_{a c} L_{b c}\right) \\
& w_{20} \quad \chi_{: a} \chi_{: a} E \quad 0 \\
& w_{21} \quad \chi_{: a a} E \quad-(m-3) F \chi_{: a a} E-(m-3) F \chi_{: a} E_{: a} \\
& w_{22} \quad \chi_{: a} \chi_{: a} \tau \quad-2(m-1) \chi_{: a} \chi_{: a} F_{; m m}+2(m-1) \chi_{: a} \chi_{: a} L_{c c} F_{; m} \\
& -4(m-1) \chi_{: a b} \chi_{: a b} F-4(m-1) \chi_{: b b a} \chi_{: a} F \\
& -16(m-1) \chi_{: a} \chi_{: b} \Omega_{a b} F+8(m-1) \chi_{: a} \Omega_{a b: b} F \\
& +4(m-1) \chi_{: a} \chi_{: b} F\left(R_{m b a m}-\rho_{a b}-L_{a b} L_{c c}+L_{a c} L_{b c}\right) \\
& w_{23} \quad \chi_{: a} \chi_{: a} \rho_{m m} \quad-(m-1) \chi_{: a} \chi_{: a} F_{; m m}+\chi_{: a} \chi_{: a} L_{c c} F_{; m}-2 \chi_{: a b} \chi_{: a b} F \\
& -2 \chi_{: b b a} \chi_{: a} F-8 \chi_{: a} \chi_{: b} \Omega_{a b} F+4 \chi_{: a} \Omega_{a b: b} F \\
& +2 F \chi_{: a} \chi_{: b}\left(-\rho_{a b}+R_{m b a m}-L_{a b} L_{c c}+L_{a c} L_{b c}\right)
\end{aligned}
$$




$$
\begin{aligned}
& w_{24} \quad \chi_{: a} \chi_{: a} \rho_{a b} \quad-\chi_{: a} \chi_{: a} F_{; m m}+\chi_{: a} \chi_{: a} L_{c c} F_{; m}+(m-2) \chi_{: a} \chi_{: b} L_{a b} F_{; m} \\
& -m \chi_{: a b} \chi_{: a b} F+2(1-m) \chi_{: a a b} \chi_{: b} F \\
& -(2 m+4) F \chi_{: a} \chi_{: b} \Omega_{a b}-(m-2) \chi_{: a a} \chi_{: b b} F \\
& +4 F \chi \chi_{: a} \Omega_{a b: b}-(m-2) F \Omega_{a b} \Omega_{a b} \\
& +(m-2) F \chi \Omega_{a b} \chi \Omega_{a b} \\
& +m F \chi_{: a} \chi_{: b}\left[-\rho_{a b}+R_{m b a m}-L_{a b} L_{c c}+L_{a c} L_{b c}\right] \\
& w_{25} \quad \chi_{: a} \chi_{: b} R_{m a b m} \quad-\chi_{: a} \chi_{: a} F_{; m m}+\chi_{: a} \chi_{: b} L_{a b} F_{; m}-2 F \chi_{: a a b} \chi_{: b} \\
& -2 F \chi_{: a} \chi_{: b} \Omega_{a b}-F \chi_{: a b} \chi_{: a b}-F \chi_{: a a} \chi_{: b b}-F \Omega_{a b} \Omega_{a b} \\
& +F \chi \Omega_{a b} \chi \Omega_{a b}+F \chi_{: a} \chi_{: b}\left[-\rho_{a b}+R_{m b a m}-L_{a b} L_{c c}\right. \\
& \left.+L_{a c} L_{b c}\right] \\
& w_{26} \quad \chi_{: a} \chi_{: a} L_{b b} L_{c c} \quad-2(m-1) F_{; m} L_{c c} \chi_{: a} \chi_{: a} \\
& w_{27} \quad \chi_{: a} \chi_{: b} L_{a c} L_{b c} \quad-2 \chi_{: a} \chi_{: b} L_{a b} F_{; m} \\
& w_{28} \quad \chi_{: a} \chi_{: a} L_{c d} L_{c d} \quad-2 F_{; m} L_{c c} \chi_{: a} \chi_{: a} \\
& w_{29} \quad \chi_{: a} \chi_{: b} L_{a b} L_{c c} \quad-\chi_{: a} \chi_{: a} L_{c c} F_{; m}-(m-1) \chi_{: a} \chi_{: b} L_{a b} F_{; m} \\
& w_{30} \quad \chi_{: a} S_{: a} L_{b b} \quad \frac{1}{4}(m-2) F_{; m} \chi_{: a} \chi_{: a} L_{c c}-(m-1) F_{; m} \chi_{: a} S_{: a} \\
& w_{31} \quad \chi_{: a} S_{: b} L_{a b} \quad-\chi_{: a} S_{: a} F_{; m}+\frac{1}{4}(m-2) F_{; m} \chi_{: a} \chi_{: b} L_{a b} \\
& w_{34} \quad \chi_{: a a} \chi_{: b b} \quad-2(m-3) F \chi_{: a a} \chi_{: b b}-2(m-3) F \chi_{: a} \chi_{: b b a} \\
& w_{35} \quad \chi_{: a b} \chi_{: a b} \quad 4 F \chi_{: a b} \chi_{: a b}+2 F \Omega_{a b} \Omega_{a b}-2 F \chi \Omega_{a b} \chi \Omega_{a b} \\
& -2 F \chi_{: a a} \chi_{: b b}+2 F \chi_{: b} \chi_{: a a b}-4 F \chi_{: a} \chi_{: b}\left[-\rho_{a b}+R_{m b a m}\right. \\
& \left.-L_{a b} L_{c c}+L_{a c} L_{b c}\right]+12 F \chi_{: a} \chi_{: b} \Omega_{a b}-4 F \chi_{: a} \Omega_{a b: b} \\
& w_{36} \quad \chi_{: a} \chi_{: a} \chi_{: b b} \quad 0 \\
& w_{37} \quad \chi_{: b} \chi_{: a a b} \quad(m-1) F \chi_{: b} \chi_{: a a b}+(m-1) F \chi_{: b b} \chi_{: a a}
\end{aligned}
$$

\section{Appendix}

In the proof of Lemma 7.1, we used the local index theorem. We list in this appendix the terms giving non-zero contributions to $a_{5}\left(D^{[1]}\right)-a_{5}\left(D^{[0]}\right)$. Coefficients of corresponding invariants are given an the right hand side in the formulae below. Let a comma denote ordinary partial differentiation. The terms with $f_{, m} f_{, m m m}$ are dropped since they are not needed for the calculations. We omit the factor of $\operatorname{Tr}_{V} I_{V}$.

$$
\begin{array}{rll}
\frac{\text { Coef }}{1440} & \frac{\text { Term }}{E_{; m} S} & \\
& & \frac{1}{2}(m-1)^{2}\left(\frac{1}{2}\left(m^{2}-8\right) f_{, m}^{2} f_{, m m}\right. \\
480 & \tau S^{2} & \frac{1}{2}(m-1)_{, a m} f_{, a m}-\frac{1}{2}\left(m^{2}-4\right) f_{, m}^{4}\left(-2 f_{, m m}-(m-2) f_{, m}^{2}\right) \\
270 & \tau_{; m} S & (m-1)^{2}\left(f_{, a m} f_{, a m}-(m-4) f_{, m m} f_{, m}^{2}+(m-2) f_{, m}^{4}\right) \\
120 & \rho_{m m} S^{2} & -\frac{1}{2}(m-1)^{3} f_{, m}^{2} f_{, m m} \\
1080 & S S_{: a a} & -\frac{1}{2}(m-1)^{2}\left(f_{, a m} f_{, a m}+\frac{1}{2} m^{2}(m-1) f_{, m}^{4}\right) \\
2880 & E S^{2} & \frac{1}{2}(m-1)^{3}\left(f_{, m}^{2} f_{, m m}+\frac{1}{4}\left(m^{2}-4\right) f_{, m}^{4}\right) \\
1440 & S^{4} & \frac{1}{2}(m-1)^{4} f_{, m}^{4}
\end{array}
$$




$$
\begin{aligned}
& \underline{\text { Coef }} \underline{\text { Term }} \\
& 270 L_{a a} E_{\text {; }} \\
& -(m-1)^{2}\left(2(m-3) f_{, m}^{2} f_{, m m}-2(m-2) f_{, m}^{4}\right. \\
& \left.-f_{\text {,am }} f_{\text {,am }}\right) \\
& 270 L_{b b} S_{: a a} \\
& \frac{1}{2}(m-1)^{2} f_{, m a} f_{, m a} \\
& 1440 L_{b b} S E \\
& -\frac{1}{2}(m-1)^{3}\left(f_{, m}^{2} f_{, m m}+\frac{1}{4}\left(m^{2}-4\right) f_{, m}^{4}\right) \\
& 30 L_{b b} S \rho_{m m} \\
& \frac{1}{2}(m-1)^{3} f_{, m}^{2} f_{, m m} \\
& \frac{1}{2}(m-1)^{3}\left(2 f_{, m}^{2} f_{, m m}+(m-2) f_{, m}^{4}\right) \\
& 240 \quad L_{b b} S \tau \\
& \text {-60 } L_{a b} \rho_{a b} S \\
& -\frac{1}{2}(m-1)^{2}\left(-f_{, m}^{2} f_{, m m}+(2-m) f_{, m}^{4}\right) \\
& 180 L_{a b} S R_{a m m b} \\
& \frac{1}{2}(m-1)^{2} f_{, m}^{2} f_{, m m} \\
& (m-1)^{3}\left(f_{, m}^{2} f_{, m m}+(m-2) f_{, m}^{4}\right) \\
& 90 L_{a b} L_{a b} E \\
& 2160 L_{b b} S^{3} \\
& 1080 L_{a a} L_{b b} S^{2} \\
& (m-1)^{2}\left(f_{, m}^{2} f_{, m m}+(m-2) f_{, m}^{4}\right) \\
& -\frac{1}{2}(m-1)^{4} f_{, m}^{4} \\
& \frac{1}{2}(m-1)^{4} f_{, m}^{4} \\
& \frac{1}{2}(m-1)^{3} f_{, m}^{4} \\
& 360 L_{a b} L_{a b} S^{2} \\
& \frac{885}{4} L_{a a} L_{b b} L_{c c} S \\
& -\frac{1}{2}(m-1)^{4} f_{, m}^{4} \\
& \frac{315}{2} \quad L_{c c} L_{a b} L_{a b} S \quad-\frac{1}{2}(m-1)^{3} f_{, m}^{4} \\
& 150 \quad L_{a b} L_{b c} L_{c a} S \quad-\frac{1}{2}(m-1)^{2} f_{, m}^{4} \\
& w_{12} \quad \chi \chi_{: a} \Omega_{a m} L_{b b} \quad 2(m-1)^{3} f_{, m}^{2} f_{, m m} \\
& w_{13} \quad \chi_{: a} \chi_{: b} \Omega_{a b} \quad 2(m-1)^{2}(m-2)\left(m^{2}+(m-2)^{2}\right) f_{, m}^{4} \\
& 90 \chi \chi: a \Omega_{a m ; m} \quad 2(m-1)\left(2(m-1) f_{, m}^{2} f_{, m m}+f_{, a m} f_{, a m}\right) \\
& 120 \chi \chi: a \Omega_{a b: b} \\
& \frac{1}{2}(m-2)\left(\left(m^{2}-(m-2)^{2}\right) f_{, a m} f_{, a m}\right. \\
& \left.+(m-1)\left(m^{4}-(m-2)^{4}\right) f_{, m}^{4}\right) \\
& w_{17} \quad \chi \chi_{: a} \Omega_{b m} L_{a b} \\
& 2(m-1)^{2} f_{, m}^{2} f_{, m m} \\
& -180 \chi_{: a} \chi_{: a} E \quad(m-1)^{2}\left(m^{2} f_{, m}^{2} f_{, m m}+\frac{1}{4}\left(m^{2}\left(m^{2}-4\right)\right.\right. \\
& \left.\left.-(m-2)^{4}\right) f_{, m}^{4}\right) \\
& -30 \chi_{: a} \chi_{: a} \tau \quad-4(m-1)^{3}\left(2 f_{, m}^{2} f_{, m m}+(m-2) f_{, m}^{4}\right) \\
& 0 \quad \chi_{: a} \chi_{: a} \rho_{m m} \quad-4(m-1)^{3} f_{, m}^{2} f_{, m m} \\
& -60 \chi_{: a} \chi_{: b} \rho_{a b} \quad-4(m-1)^{2}\left(f_{, m}^{2} f_{, m m}+(m-2) f_{, m}^{4}\right) \\
& 30 \chi_{: a} \chi_{: b} R_{m a b m}-4(m-1)^{2} f_{, m}^{2} f_{, m m} \\
& \begin{array}{rll}
\frac{255}{4} & \chi_{: a a} \chi_{: b b} & (m-1)\left(4 f_{, a m} f_{, a m}+(m-1)\left(m^{4}-(m-2)^{4}\right) f_{, m}^{4}\right) \\
-\frac{105}{2} & \chi_{: a b} \chi_{: a b} & (m-1)\left(4(m-1) f_{, a m} f_{, a m}+\left(m^{4}-(m-2)^{4}\right) f_{, m}^{4}\right) \\
\underline{15} & \chi_{a, a} \chi_{: a} \chi_{: b} \chi_{: b} & (m-1)^{2}\left(m^{4}-(m)^{4}\right) f^{4}
\end{array} \\
& \frac{15}{4} \quad \chi_{: a} \chi_{: a} \chi_{: b} \chi_{: b} \quad(m-1)^{2}\left(m^{4}-(m-2)^{4}\right) f_{, m}^{4} \\
& \begin{array}{rll}
\frac{15}{8} & \chi_{: a} \chi_{: b} \chi_{: a} \chi_{: b} & -(m-1)(m \\
-\frac{675}{8} & \chi_{: a} \chi_{: b} L_{a b} L_{c c} & 4(m-1)^{3} f_{, m}^{4}
\end{array} \\
& -\frac{675}{32} \quad \chi_{: a} \chi_{: a} L_{b b} L_{c c} \quad 4(m-1)^{4} f_{, m}^{4} \\
& -\frac{75}{4} \quad \chi_{: a} \chi_{: b} L_{a c} L_{c b} \quad 4(m-1)^{2} f_{, m}^{4} \\
& -\frac{195}{16} \quad \chi_{: a} \chi_{: a} L_{c d} L_{c d} \quad 4(m-1)^{3} f_{, m}^{4} \\
& -330 \quad \chi_{: a} S_{: a} L_{b b} \quad-\frac{1}{2} m^{2}(m-1)^{3} f_{, m}^{4} \\
& -300 \quad \chi_{: a} S_{: b} L_{a b} \quad-\frac{1}{2} m^{2}(m-1)^{2} f_{, m}^{4} \\
& 720 \chi S_{: a} S_{: a} \quad \frac{1}{2}(m-1)^{2} f_{, a m} f_{, a m}
\end{aligned}
$$




$$
\begin{array}{rll}
\frac{\text { Coef }}{0} & \frac{\text { Term }}{\Omega_{a b} \Omega_{a b}+\chi \Omega_{a b} \chi \Omega_{a b}} & -\frac{1}{2}(m-1)(m-2)\left(m^{4}-(m-2)^{4}\right) f_{, m}^{4} \\
\frac{1}{2}\left(w_{5}-w_{7}\right) & \Omega_{a b} \Omega_{a b}-\chi \Omega_{a b} \chi \Omega_{a b} & -4(m-1)(m-2) f_{, a m} f_{, a m} \\
-45 & \Omega_{a m} \Omega_{a m}+\chi \Omega_{a m} \chi \Omega_{a m} & -2(m-1)(m-2) f_{, a m} f_{, a m} \\
-360 & \Omega_{a m} \chi S_{: a}-\Omega_{a m} S_{: a} \chi & -\frac{1}{2} m^{2}(m-1)^{2} f_{, m}^{2} f_{, m m}
\end{array}
$$

\section{References}

[1] T.P. Branson and P.B. Gilkey, The asymptotics of the Laplacian on a manifold with boundary, Commun. Partial Diff. Eq. 15 (1990), 245-272.

[2] —, Residues of the eta function for an operator of Dirac type with local boundary conditions, Diff. Geom. and its Applications 2 (1992), 249267.

[3] T.P. Branson, P.B. Gilkey and D.V. Vassilevich, The asymptotics of the Laplacian on a manifold with boundary II, Boll. Unione Mat. Ital. (7) 11B Suppl. Fasc. 2 (1997), 39-67, hep-th/9504029.

[4] T.P. Branson, P.B. Gilkey and D.V. Vassilevich, Vacuum expectation value asymptotics for second order differential operators on manifolds with boundary, J. Math. Phys. 39 (1998), 1040-1049.

[5] J.S. Dowker, J.S. Apps, K. Kirsten and M. Bordag, Spectral invariants for the Dirac operator on the d ball with various boundary conditions, Class. Quantum Grav. 13 (1996), 2911-2920.

[6] J.S. Dowker and K. Kirsten, Smeared heat-kernel coefficients on the ball and generalized cone, hep-th/9803094.

[7] J.S. Dowker and K. Kirsten, Spinors and forms on the ball and the generalized cone, Communications in Analysis and Geometry 7 (1999), 451-489.

[8] E. Elizalde, M. Lygren and D.V. Vassilevich, Zeta function for the Laplace operator acting on forms in a ball with gauge boundary conditions, Commun. Math. Phys. 183 (1997), 645-660.

[9] G. Esposito, A. Kamenshchik and G. Pollifrone, Euclidean Quantum Gravity on Manifolds with Boundary, Kluwer, 1997 ISBN: 0-7923-44723.

[10] G. Kennedy, R. Critchley, and J.S. Dowker, Finite temperature field theory with boundaries: stress tensor and surface action renormalization, Ann. Phys. 125 (1980), 346-400. 
[11] P. Greiner, An asymptotic expansion for the heat equation, Arch. Rat. Mech. Anal. 41 (1971), 163-218.

[12] G. Grubb, Functional Calculus of Pseudo-differential Boundary Problems, Progress in Math. 65, Birkhäuser 1986.

[13] K. Kirsten, The $a_{5}$ heat kernel coefficient on a manifold with boundary, Class. Quantum Grav. 15 (1998), L5-L12.

[14] H.P. McKean and I.M.Singer, Curvature and eigenvalues of the Laplacian, J. Diff. Geo. 1 (1967), 43-69.

[15] I. Moss and J.S. Dowker, The correct $B_{4}$ coefficient, Phys. Letters B 229 (1989), 261-263.

[16] R.T. Seeley, Singular integrals and boundary problems, Amer. J. Math. 88 (1966), 781-809.

[17] E. Sezgin, Topics in M-theory, Contribution to the Abdus Salam Memorial Meeting, hep-th/9809204.

[18] D.V. Vassilevich, Vector fields on a disk with mixed boundary conditions, J. Math. Phys. 36 (1995), 3174-3182.

[19] D.V. Vassilevich, Heat kernel for antisymmetric tensor field on a disk, Phys. Lett. B 348 (1995), 39-43.

T. Branson, Department of Mathematics, The University of Iowa, Iowa City IA 52242 USA. EMAIL: branson@math.uiowa.edu

P. Gilkey, Department of Mathematics, University of Oregon, Eugene OR 97403 USA. EMAIL: gilkey@darkwing.uoregon.edu

K. Kirsten, Department of Physics and Astronomy, The University of Manchester, Oxford Road, Manchester, England. EMAIL: klaus@a13.ph.man.ac.uk D. Vassilevich, Institute for Theoretical Physics, University of Leipzig, Augustusplatz 10, 04109 Leipzig, Germany. EMAIL: vassil@itp.uni-leipzig.de 